\begin{tabular}{|c|l|}
\hline Title & A scaling limit of a Hamiltonian of many nonrelativistic particles interacting with a quantized radiation field \\
\hline Author(s) & Hiroshima, F. \\
\hline Citation & Hokkaido University Preprint Series in Mathematics, 338, 1-34 \\
\hline Issue Date & 1996-5-1 \\
\hline DOI & 10.14943/83484 \\
\hline Doc URL & http://hdl.handle.net/2115/69088 \\
\hline Type & bulletin (article) \\
\hline File Information & pre338.pdf \\
\hline
\end{tabular}

Instructions for use 
A Scaling limit of a Hamiltonian of many nonrelativistic particles interacting with a quantized radiation field

Fumio Hiroshima

Series \#338. May 1996 


\section{HOKKAIDO UNIVERSITY PREPRINT SERIES IN MATHEMATICS}

\#314 T. Mikami, Weak convergence on the first exit time of randomly perturbed dynamical systems with a repulsive equilibrium point, 20 pages. 1995.

\#315 A. Arai, Canonical commutation relations, the Weierstrass Zetafunction, and infinite dimensional Hilbert space representations of the quantum group $U_{q}\left(\mathfrak{s l}_{2}\right), 22$ pages. 1995.

\#316 Y. Shibukawa, Vertex-face correspondence in elliptic solutions of the Yang-Baxter equation, 8 pages. 1995.

\#317 M.-H. Giga and Y. Giga, Consistency in evolutions by crystalline curvature, 16 pages. 1995.

\#318 Wei-Zhi Sun, Shadows of moving surfaces, 19 pages. 1995.

\#319 S. Izumiya and G.T. Kossioris, Bifurcations of shock waves for viscosity solutions of Hamilton-Jacobi equations of one space variable, 39 pages. 1995 .

\#320 T. Teruya, Normal intermediate subfactors, 44 pages. 1995.

\#321 M. Ohnuma, Axisymmetric solutions and singular parabolic equations in the theory of viscosity solutions, 26 pages. 1995.

\#322 T. Nakazi, An outer function and several important functions in two variables, 12 pages. 1995.

\#323 N. Kawazumi, An infinitesimal approach to the stable cohomology of the moduli of Riemann surfaces, 22 pages. 1995.

\#324 A. Arai, Factorization of self-adjoint operators by abstract Dirac operators and its application to second quantizations on Boson Fermion Fock spaces, 15 pages. 1995.

\#325 K. Sugano, On strongly separable Frobenius extensions, 11 pages. 1995.

\#326 D. Lehmann and T. Suwa, Residues of holomorphic vector fields on singular varieties, 21 pages. 1995.

\#327 K. Tsutaya, Local regularity of non-resonant nonlinear wave equations, 23 pages. 1996.

\#328 T. Ozawa and Y. Tsutsumi, Space-time estimates for null gauge forms and nonlinear Schrödinger equations, 25 pages. 1996.

\#329 O. Ogurisu, Anticommutativity and spin 1/2 Schrödinger operators with magnetic fields, 12 pages. 1996.

\#330 Y. Kurokawa, Singularities for projections of contour lines of surfaces onto planes, 24 pages. 1996.

\#331 M.-H. Giga and Y. Giga, Evolving graphs by singular weighted curvature, 94 pages. 1996.

\#332 M. Ohnuma and K. Sato, Singular degenerate parabolic equations with applications to the $p$-laplace diffusion equation, 20 pages. 1996.

\#333 T. Nakazi, The spectra of Toeplitz operators with unimodular symbols, 9 pages. 1996.

\#334 B. Khanedani and T. Suwa, First variation of horomorphic forms and some applications, 11 pages. 1996.

\#335 J. Seade and T. Suwa, Residues and topological invariants of singular holomorphic foliations ${ }^{1}, 28$ pages. 1996.

\#336 Y. Giga, M.E. Gurtin and J. Matias, On the dynamics of crystalline motions, 67 pages. 1996.

\#337 I. Tsuda, A new type of self-organization associated with chaotic dynamics in neural networks, 22 pages. 1996. 


\title{
A Scaling limit of a Hamiltonian of many nonrelativistic particles interacting with a quantized radiation field
}

\author{
Fumio HIROSHIMA \\ Department of Mathematics, Faculty of Science, Hokkaido University \\ Sapporo 060 Japan
}

\begin{abstract}
This paper presents a scaling limit of Hamiltonians which describe interactions of $N$-nonrelativistic charged particles in a scalar potential and a quantized radiation field in the Coulomb gauge with the dipole approximation. The scaling limit defines effective potentials. In one-nonrelativistic particle case, the effective potentials have been known to be Gaussian transformations of the scalar potential [J.Math.Phys.34(1993)44784518]. However it is shown that the effective potentials in the case of $N$-nonrelativistic particles are not necessary to be Gaussian transformations of the scalar potential.
\end{abstract}




\section{INTRODUCTION}

The main problem in this paper is to consider a scaling limit of a model in quantum electrodynamics which describes an interaction of many nonrelativistic charged particles and a quantized radiation field in the Coulomb gauge with the dipole approximation. For our discussion we may limit ourselves to the case of a fixed number $N$ of the particles, since $N$ dose not change in time. The model we consider is called "the Pauli-Fierz model", which has been a subject of great interests and by which real physical phenomena of charged particles and a quantized radiation field such as "Lamb shift" can be interpreted. There has been a considerable amount of literature on the Pauli-Fierz model with one-nonrelativistic charged particle, e.g., $[1,2]$ from points of view of physics and $[3,4,5,6,7,8]$ mathematical points of view. In particular, the authors of $[5,6]$ have studied a scaling limit of the Pauli-Fierz model with one-nonrelativistic charged particle. We may well extend the scaling limit of one-particle system to $N$-particle system.

The authors of $[5,6]$ defined Hamiltonians of the Pauli-Fierz model as self-adjoint operators $H_{\rho}$ with an ultraviolet cut-off function $\rho$ acting in the tensor product of the Hilbert space $L^{2}\left(\mathbb{R}^{d}\right)$ and a Boson Fock space $\mathcal{F}(\mathcal{W})$ over $\mathcal{W}=\Theta_{r=1}^{d-1} L^{2}\left(\mathbb{R}^{d}\right)$. Introducing scalings with respect to parameters $c$ (the speed of light), $m$ (the mass of the particle) and $e$ (the charge of the particle), the authors have shown the existence of the strong resolvent limits of the scaled self-adjoint operators $H_{\rho}^{R E N}(\kappa)+V \otimes I$ with an infinite self-energy of the nonrelativistic particle subtracted with a scalar potential $V$, (we call the limit "the scaling limit of $\left.H_{\rho}+V \otimes I "\right):$ In [6] we have proved the following:

Let $V$ and $\rho$ satisfy some conditions and $\Delta$ be the Laplacian in $L^{2}\left(\mathbb{R}^{d}\right)$. Then $H_{\rho}^{R E N}(\kappa)+$ $V \otimes I$ is self-adjoint and bounded from below uniformly in sufficiently large $\kappa>0$ with

$$
s-\lim _{\kappa \rightarrow \infty}\left(H_{\rho}^{R E N}(\kappa)+V \otimes I-z\right)^{-1}=\mathcal{S}\left\{\left(-\frac{1}{2 m_{\infty}} \Delta+V_{\text {eff }}-z\right)^{-1} \otimes P_{0}\right\} \mathcal{S}^{-1},
$$

where $z \in \mathbb{C} \backslash \mathbb{R}, m_{\infty}$ is a positive constant, $\mathcal{S}$ a unitary operator on $L^{2}\left(\mathbb{R}^{d}\right) \otimes \mathcal{F}(\mathcal{W}), P_{0}$ a 
projection on $\mathcal{F}(\mathcal{W})$ and $V_{\text {eff }}$ a multiplication operator defined by

$$
V_{e f f}(x)=(2 \pi \alpha)^{-\frac{d}{2}} \int d y e^{-|x-y|^{2} / 2 \alpha} V(y)
$$

where $\alpha$ is a positive constant. The multiplication operator $V_{\text {eff }}$ is called "the effective potential".

One of the strongest methods to analyze the scaling limits in $[5,6]$ was to find Bogoliubov transformations $\mathcal{U}$, which implements a unitary equivalence between the Pauli-Fierz Hamiltonians $H_{\rho}$ and decoupled Hamiltonians of the form

$$
\widetilde{H}=-\frac{1}{2 \widetilde{m}} \Delta \otimes I+I \otimes H_{b}+\text { constant }
$$

where $\widetilde{m}$ is a positive constant and $H_{b}$ is the free Hamiltonian of the quantized radiation field in $\mathcal{F}(\mathcal{W})$; the authors of $[5,6]$ show equations of the following type:

$$
\left(H_{\rho}^{R E N}+V \otimes I-z\right)^{-1}=\mathcal{U}\left(\widetilde{H}+\mathcal{U}^{-1}(V \otimes I) \mathcal{U}-z\right)^{-1} \mathcal{U}^{-1}
$$

In this paper, the Pauli-Fierz Hamiltonian $H_{\vec{p}}$ with $N$-nonrelativistic charged particles in the Coulomb gauge with the dipole approximation are defined as operators acting in the Hilbert space $\underbrace{L^{2}\left(\mathbb{R}^{d}\right) \otimes \ldots \otimes L^{2}\left(\mathbb{R}^{d}\right)}_{N} \otimes \mathcal{F}(\mathcal{W}) \cong L^{2}\left(\mathbb{R}^{d N}\right) \otimes \mathcal{F}(\mathcal{W})$ by

$$
\begin{aligned}
H_{\vec{\rho}} & =\frac{1}{2 m} \sum_{j=1}^{N} \sum_{\mu=1}^{d}\left(-i \hbar D_{\mu}^{j} \otimes I-e I \otimes A_{\mu}\left(\rho_{j}\right)\right)^{2}+I \otimes H_{b} \\
& =-\frac{\hbar^{2}}{2 m} \Delta \otimes I+I \otimes H_{b}+\frac{1}{2 m} \sum_{j=1}^{N} \sum_{\mu=1}^{d}\left(2 e \hbar i D_{\mu}^{j} \otimes A_{\mu}\left(\rho_{j}\right)+e^{2} I \otimes A_{\mu}^{2}\left(\rho_{j}\right)\right),
\end{aligned}
$$

where $D_{\mu}^{j}$ is the differential operator with respect to the $j$-th variable in the $\mu$-th direction, $\Delta$ the Laplacian in $L^{2}\left(\mathbb{R}^{d N}\right), \hbar$ the Planck constant divided $2 \pi$ and $A_{\mu}\left(\rho_{j}\right)$ the quantized radiation field in the $\mu$-th direction with an ultraviolet cut-off function $\rho_{j}$ in the Coulomb gauge. Problems arising in the many particles system are as follows:

(i) Do there any Bogoliubov transformations such as (1.1) exist? 
(ii) What kind of scalar potentials $V$ and sets of ultraviolet cut-off functions $\left(\rho_{1}, \ldots, \rho_{N}\right)$ do a scaling limit of the Hamiltonian $H_{\vec{p}}+V \otimes I$ exist for ? Furthermore, what kind of infinite self-energy should be subtracted from the original Hamiltonian $H_{\vec{\rho}}+V \otimes I$ ?

(iii) If the scaling limit exists, what form does the effective potential have?

With this motivation, we continue here to analyze a scaling limit of the Pauli-Fierz model with $N$-nonrelativistic charged particles.

We introduce the same scaling as [6] as follows;

$$
c(\kappa)=c \kappa, e(\kappa)=e \kappa^{-\frac{1}{2}}, m(\kappa)=m \kappa^{-2}
$$

Introducing a pseudo differential operator $E^{R E N}(D, \kappa)$ in $L^{2}\left(\mathbb{R}^{d N}\right)$ with a symbol $E^{R E N}(p, \kappa)$ such that $E^{R E N}(p, \kappa) \rightarrow \infty$ as $\kappa \rightarrow \infty$, we define a Hamiltonian $H_{\vec{\rho}}^{R E N}(\kappa)$ by $H_{\vec{\rho}}^{R E N}(\kappa)=-E^{R E N}(D, \kappa) \otimes I+\kappa I \otimes H_{b}+\frac{1}{2 m} \sum_{j=1}^{N} \sum_{\mu=1}^{d}\left(\kappa 2 e \hbar i D_{\mu}^{j} \otimes A_{\mu}\left(\rho_{j}\right)+e^{2} I \otimes A_{\mu}^{2}\left(\rho_{j}\right)\right)$.

For sufficiently large $\kappa>0$ and a scalar potential $V$ with some conditions, we shall show that $H_{\vec{\rho}}^{R E N}(\kappa)+V \otimes I$ is essentially self-adjoint on $D(-\Delta \otimes I) \cap D\left(I \otimes H_{b}\right)$ and bounded from below uniformly in sufficiently large $\kappa>0$, and the existence of Bogoliubov transformations $\mathcal{U}(\kappa)$, which gives a unitary equivalence of $H_{\vec{\rho}}^{R E N}(\kappa)+V \otimes I$ and a self-adjoint operator $\widetilde{H_{\vec{\rho}}}(\kappa)+C_{\kappa}(V)$ as follows;

$$
\left(H_{\vec{\rho}}^{R E N}(\kappa)+V \otimes I-z\right)^{-1}=\mathcal{U}(\kappa)\left(\widetilde{H_{\vec{\rho}}}(\kappa)+C_{\kappa}(V)-z\right)^{-1} \mathcal{U}^{-1}(\kappa)
$$

where $\widetilde{H_{\vec{\rho}}}(\kappa)=\tilde{E}(D, \kappa) \otimes I+\kappa I \otimes H_{b}, \tilde{E}(D, \kappa)$ is a pseudo differential operator in $L^{2}\left(\mathbb{R}^{d N}\right)$ and $C_{\kappa}(V)=\mathcal{U}^{-1}(\kappa)(V \otimes I) \mathcal{U}(\kappa)($ Theorem 3.5). Then we see that $\mathcal{U}(\kappa) \rightarrow \mathcal{U}(\infty)$ as $\kappa \rightarrow \infty$ strongly (Theorem 3.4) and hence we get

$$
s-\lim _{\kappa \rightarrow \infty}\left(H_{\vec{\rho}}^{R E N}(\kappa)+V \otimes I-z\right)^{-1}=\mathcal{U}(\infty)\left\{\left(E^{\infty}(D)+V_{e f f}-z\right)^{-1} \otimes P_{0}\right\} \mathcal{U}^{-1}(\infty)
$$


where $E^{\infty}(D)$ is a pseudo differential operator in $L^{2}\left(\mathbb{R}^{d N}\right)$ and $V_{e f f}$ a multiplication operator. (Theorems $3.6,3.7$ ). In the case of one-particle system the effective potential $V_{\text {eff }}$ is a Gaussian transformation of a given scalar potential $V$. However, we shall see that in the $N$-particle system, $V_{\text {eff }}$ is not necessary to be a Gaussian transformation. Actually it is determined by a matrix $\tilde{\Delta}^{\infty}=\left(\tilde{\Delta}_{i j}^{\infty}\right)_{1 \leq i, j \leq N}$ defined by

$$
\tilde{\Delta}_{i j}^{\infty}=\frac{1}{2} \frac{d-1}{d}\left(\frac{\hbar}{m c}\right) \frac{e^{2}}{\hbar c} \int_{\mathbb{R}^{d}} d k \frac{\hat{\rho}_{i}(k) \hat{\rho}_{j}(k)}{\omega(k)^{3}},
$$

where $\omega(k)=|k|, k \in \mathbb{R}^{d}$. In the case where $\tilde{\Delta}^{\infty}$ is non-degenerate, the effective potential $V_{e f f}$ is Gaussian transformations of $V$.

The outline of this paper is as follows. In section 2, we define the Pauli-Fierz Hamiltonian with $N$-nonrelativistic charged particles in the Coulomb gauge with the dipole approximation and show its self-adjointness. Moreover we construct an exact solution to the Heisenberg equation from the point of view of the operator theory (Corollary 2.9). In section 3, when the scaling parameter $\kappa>0$ is sufficiently large, we show that a Bogoliubov transformation can be constructed, and define a renormalized self-adjoint operator $H_{\vec{\rho}}^{R E N}(\kappa)$ which is the original Hamiltonian $H_{\vec{\rho}}(\kappa)$ with an infinite self-energy of the nonrelativistic charged particles subtracted. We shall show the existence of the scaling limit of $H_{\vec{\rho}}+V \otimes I$ and give an explicit form of the effective potential. We give a typical example of a scalar potential and a set of ultraviolet cut-off functions. In section 4, we give a physical interpretation of the matrix $\tilde{\Delta}^{\infty}$.

The author would like to thank Professor A.Arai for helpful discussions.

\section{THE PAULI-FIERZ MODEL AND EXACT SO- LUTION}

To begin with, let us introduce some preliminary notations. Let $\mathcal{H}$ be a Hilbert space over $\mathbb{C}$. We denote the inner product and the associated norm by $\langle *, \cdot\rangle_{\mathcal{H}}$ and $\|\cdot\|_{\mathcal{H}}$ respectively. 
The inner product is linear in and antilinear in $*$. The domain of an operator $A$ in $\mathcal{H}$ is denoted by $D(A)$. A notation The Fourier transformation of a function $f$ is denoted by $\hat{f}$ (resp. $\check{f}$ ) and $\bar{f}$ the complex conjugate of $f$. In this paper, summations over repeated Greek letters are understood. Let

$$
\mathcal{W} \equiv \underbrace{L^{2}\left(\mathbb{R}^{d}\right) \oplus \ldots \oplus L^{2}\left(\mathbb{R}^{d}\right)}_{d-1}
$$

We define the Boson Fock space over $\mathcal{W}$ by

$$
\mathcal{F}(\mathcal{W}) \equiv \bigoplus_{n=0}^{\infty} \otimes_{s}^{n} \mathcal{W} \equiv \bigoplus \mathcal{F}_{n}(\mathcal{W})
$$

where $\otimes_{s}^{0} \mathcal{W} \equiv \mathbb{C}$ and $\otimes_{s}^{n} \mathcal{W}(n \geq 1)$ denotes the $\mathrm{n}$-fold symmetric tensor product. Put

$$
\mathcal{F}^{N}(\mathcal{W}) \equiv \bigoplus_{n=0}^{N} \mathcal{F}_{n}(\mathcal{W}) \underset{n \geq N+1}{\bigoplus_{n}}\{0\}
$$

Moreover we define the finite particle subspace of $\mathcal{F}(\mathcal{W})$ by

$$
\mathcal{F}^{\infty}(\mathcal{W}) \equiv \bigcup_{N=0}^{\infty} \mathcal{F}^{N}(\mathcal{W})
$$

The annihilation operator $a(f)$ and the creation operator $a^{\dagger}(f)(f \in \mathcal{W})$ act on the finite particle subspace and leave it invariant with the canonical commutation relations (CCR): for $f, g \in \mathcal{W}$

$$
\begin{aligned}
{\left[a(f), a^{\dagger}(g)\right] } & =\langle\bar{f}, g\rangle_{\mathcal{W}}, \\
{\left[a^{\sharp}(f), a^{\sharp}(g)\right] } & =0,
\end{aligned}
$$

where $[A, B]=A B-B A, a^{\sharp}$ denotes either $a$ or $a^{\dagger}$. Furthermore,

$$
\left\langle a^{\dagger}(f) \Phi, \Psi\right\rangle_{\mathcal{F}(\mathcal{W})}=\langle\Phi, a(\bar{f}) \Psi\rangle_{\mathcal{F}(\mathcal{W})}, \quad \Phi, \Psi \in \mathcal{F}^{\infty}(\mathcal{W})
$$

We define polarization vectors $e^{r}(r=1, \ldots, d-1)$ as measurable functions $e^{r}: \mathbb{R}^{d} \longrightarrow \mathbb{R}^{d}$ such that

$$
e^{r}(k) e^{s}(k)=\delta_{r s}, \quad e^{r}(k) k=0, \quad \text { a.e. } k \in \mathbb{R}^{d}
$$


In this paper, we fix polarization vectors $e^{r}$. The $\mu$-th direction time-zero smeared radiation field in the Coulomb gauge with the dipole approximation is defined as operators acting in $\mathcal{F}(\mathcal{W})$ by

$$
A_{\mu}(f)=\frac{1}{\sqrt{2}}\left\{a^{\dagger}\left(\oplus_{r=1}^{d-1} \frac{\sqrt{\hbar} e_{\mu}^{r} \hat{f}}{\sqrt{c \omega}}\right)+a\left(\oplus_{r=1}^{d-1} \frac{\sqrt{\hbar} e_{\mu}^{r} \tilde{\hat{f}}}{\sqrt{c \omega}}\right)\right\}
$$

and the conjugate momentum

$$
\Pi_{\mu}(f)=\frac{i}{\sqrt{2}}\left\{a^{\dagger}\left(\oplus_{r=1}^{d-1} \sqrt{\hbar} \sqrt{\omega \omega} e_{\mu}^{r} \hat{f}\right)-a\left(\oplus_{r=1}^{d-1} \sqrt{\hbar} \sqrt{c \omega} e_{\mu}^{r} \hat{\hat{f}}\right)\right\}
$$

where $\tilde{g}(k)=g(-k)$. Note that in the case where $f$ is real-valued, $A_{\mu}(f)$ and $\Pi_{\mu}(f)$ are symmetric operators. Let $\Omega=(1,0,0, \ldots) \in \mathcal{F}(\mathcal{W})$. It is well known that

$$
\mathcal{L}\left\{a^{\dagger}\left(f_{1}\right) \ldots a^{\dagger}\left(f_{n}\right) \Omega, \Omega \mid f_{j} \in \mathcal{W}, j=1, \ldots, n, n \geq 1\right\}
$$

is dense in $\mathcal{F}(\mathcal{W})$. For a nonnegative self-adjoint operator $h: \mathcal{W} \rightarrow \mathcal{W}$, an operator $\Gamma\left(e^{-t h}\right)$ is defined by

$$
\begin{aligned}
\Gamma\left(e^{-t h}\right) a^{\dagger}\left(f_{1}\right) \ldots a^{\dagger}\left(f_{n}\right) \Omega & =a^{\dagger}\left(e^{-t h} f_{1}\right) \ldots a^{\dagger}\left(e^{-t h} f_{n}\right) \Omega \\
\Gamma\left(e^{-t h}\right) \Omega & =\Omega .
\end{aligned}
$$

The operator $\Gamma\left(e^{-t h}\right)$ defines a unique strongly continuous one-parameter semigroup on $\mathcal{F}(\mathcal{W})$. Hence, by Stone's theorem, there exists a nonnegative self-adjoint operator $d \Gamma(h)$ in $\mathcal{F}(\mathcal{W})$ such that

$$
\Gamma\left(e^{-t h}\right)=e^{-t d \Gamma(h)}
$$

The operator $d \Gamma(h)$ is called "the second quantization of $h$ ". Put $\tilde{\omega}=\underbrace{\omega \oplus \ldots \oplus \omega}_{d-1}$. The free Hamiltonian $H_{b}$ in $\mathcal{F}(\mathcal{W})$ is defined by

$$
H_{b} \equiv \hbar c d \Gamma(\widetilde{\omega})
$$


Let $M_{d}$ be a Hilbert space defined by

$$
M_{d}=\left\{\left.f\left|\int\right| f(k)\right|^{2} \omega(k)^{d} d k<\infty\right\}
$$

with the inner product

$$
<f, g>_{n}=\int_{\mathbb{R}^{d}} \bar{f}(k) g(k) \omega(k)^{n} d k
$$

We have the following commutation relations on $\mathcal{F}^{\infty}(\mathcal{W})$,

$$
\begin{array}{lll}
{\left[A_{\mu}(f), A_{\nu}(g)\right]=0,} & & \hat{f}, \hat{g} \in M_{-1}, \\
{\left[\Pi_{\mu}(f), \Pi_{\nu}(g)\right]=0,} & & \hat{f}, \hat{g} \in M_{1}, \\
{\left[A_{\mu}(f), \Pi_{\nu}(g)\right]=i \hbar\left\langle d_{\mu \nu} \hat{\bar{f}}, \hat{g}\right\rangle_{L^{2}\left(\mathbb{R}^{d}\right)},} & \hat{f}, \hat{g} \in M_{-1} \cap M_{0} \cap M_{1}
\end{array}
$$

and on $D\left(H_{b}^{\frac{3}{2}}\right)$,

$$
\begin{array}{ll}
{\left[H_{b}, A_{\mu}(f)\right]=-i \hbar \Pi_{\mu}(f),} & \hat{f} \in M_{-1} \cap M_{1}, \\
{\left[H_{b}, \Pi_{\mu}(f)\right]=i \hbar c^{2} A_{\mu}(-\Delta f),} & \hat{f} \in M_{3} \cap M_{1},
\end{array}
$$

where $\Delta$ is the Laplacian in the $L^{2}$-sense and $d_{\mu \nu}(k)=\sum_{r=1}^{d} e_{\mu}^{r}(k) e_{\nu}^{r}(k)$. The Pauli-Fierz Hamiltonian with $N$-nonrelativistic charged particles interacting with the quantized radiation field in the Coulomb gauge with the dipole approximation is defined by

$$
H_{\vec{\rho}} \equiv H_{\rho_{1}, \ldots, \rho_{N}} \equiv \frac{1}{2 m} \sum_{j=1}^{N} \sum_{\mu=1}^{d}\left(-i \hbar D_{\mu}^{j} \otimes I-e I \otimes A_{\mu}\left(\rho_{j}\right)\right)^{2}+I \otimes H_{b},
$$

acting in

$$
\underbrace{L^{2}\left(\mathbb{R}^{d}\right) \otimes \ldots \otimes L^{2}\left(\mathbb{R}^{d}\right)}_{N} \otimes \mathcal{F}(\mathcal{W}) \cong L^{2}\left(\mathbb{R}^{d N}\right) \otimes \mathcal{F}(\mathcal{W}) \cong \int_{\mathbb{R}^{d N}}^{\oplus} \mathcal{F}(\mathcal{W}) d x
$$

where $D_{\mu}^{j}$ is the $L^{2}$-derivative with respect to the $\mathrm{j}$-th variable in the $\mu$-th direction, $\rho_{j}^{\prime} s$ serve as ultraviolet cut-off functions. We introduce a scaling with respect to the parameters $c, e, m$ as (1.2). Throughout this paper, for objects $A=A(c, e, m)$ containing the parameters $c, e, m$, we denote the scaled object by $A(\kappa) \equiv A(c(\kappa), e(\kappa), m(\kappa))$. We define a class of sets of functions as follows: 
Definition $2.1 \vec{\rho}=\left(\rho_{1}, \ldots, \rho_{N}\right)$ is in $P$ if and only if

(1) $\hat{\rho}_{j}, j=1, \ldots, N$ are rotation invariant, $\hat{\rho}_{j}(k)=\hat{\rho}_{j}(|k|)$, and real-valued,

(2) $\hat{\rho}_{j} / \omega, \hat{\rho}_{j} / \sqrt{\omega}, \hat{\rho}_{j}, \sqrt{\omega} \hat{\rho}_{j} \in L^{2}\left(\mathbb{R}^{d}\right)$.

Moreover $\vec{\rho}$ is in $\widetilde{P}$ if and only if in addition to (1) and (2) above

(3) For all $j=1, \ldots, N, \hat{\rho}_{j} / \omega \sqrt{\omega} \in L^{2}\left(\mathbb{R}^{d}\right)$ and there exist $0<\alpha<1$ and $1 \leq \epsilon$ such that $\hat{\rho}_{i}(\sqrt{s}) \hat{\rho}_{j}(\sqrt{s})(\sqrt{s})^{d-2} \in \operatorname{Lip}(\alpha) \cap L^{\epsilon}([0, \infty))$, where Lip $(\alpha)$ is the set of the Lipschitz continuous functions on $[0, \infty)$ with order $\alpha$,

(4) $\sup _{k}\left|\hat{\rho}_{j}(k) \omega^{\frac{d}{2}-\frac{3}{2}}(k)\right|<\infty, \sup _{k}\left|\hat{\rho}_{j}(k) \omega^{\frac{d}{2}-\frac{1}{2}}(k)\right|<\infty, j=1, \ldots, N$.

Observe that Definition 2.1 (1) implies that $\rho_{j}$ 's are real-valued functions. Hence $A_{\mu}\left(\rho_{j}\right)^{\prime}$ 's are symmetric operators. Put

$$
H_{0}=-\frac{1}{2 m} \hbar^{2} \Delta \otimes I+I \otimes H_{b}
$$

where $\Delta$ is the Laplacian in $L^{2}\left(\mathbb{R}^{d N}\right)$. It is well known that $H_{0}$ is a nonnegative self-adjoint operator on $D\left(H_{0}\right)=D\left(-\frac{1}{2 m} \hbar^{2} \Delta \otimes I\right) \cap D\left(I \otimes H_{b}\right)$.

Theorem $2.2([3,4])$ For $\vec{\rho} \in P$ and $\kappa>0$, the operator $H_{\vec{\rho}}(\kappa)$ is self-adjoint on $D\left(H_{0}\right)$ and essentially self-adjoint on any core of $H_{0}$ and nonnegative.

Let $\mathbf{F}=F \otimes I$, where $F$ denotes the Fourier transform in $L^{2}\left(\mathbb{R}^{d N}\right)$. It is clear that operators $\mathbf{F} H_{\bar{\rho}} \mathrm{F}^{-1}$ can be decomposable as follows:

$$
\mathbf{F} H_{\vec{\rho}}(\kappa) \mathbf{F}^{-1}=\int_{\mathbb{R}^{d N}}^{\oplus} H_{\vec{\rho}}(p, \kappa) d p
$$

where

$$
H_{\vec{\rho}}(p, \kappa)=\frac{1}{2 m} \sum_{j=1}^{N} \sum_{\mu=1}^{d}\left(\kappa \hbar p_{\mu}^{j}-e A_{\mu}\left(\rho_{j}\right)\right)^{2}+\kappa H_{b}
$$


Theorem $2.3([3,4])$ For $\vec{\rho} \in P$ and $\kappa>0$, the operator $H_{\vec{\rho}}(p, \kappa)$ is self-adjoint on $D\left(H_{0}\right)$ and essentially self-adjoint on any core of $H_{b}$ and nonnegative.

Following $[3,4,6]$, we shall construct a Heisenberg field concretely. The Heisenberg field $A_{\mu}(f, t, \kappa)$ with the scaling parameter $\kappa$ is defined by a solution to the Heisenberg equation:

$$
\begin{aligned}
\frac{d}{d t} A_{\mu}(f, t, \kappa) & =\frac{i}{\hbar}\left[H_{\vec{\rho}}(p, \kappa), A_{\mu}(f, t, \kappa)\right], \\
A_{\mu}(f, 0, \kappa) & =A_{\mu}(f, \kappa) .
\end{aligned}
$$

In order to construct the Heisenberg field in a rigorous way, we shall prepare some technical lemmas. We define an $N \times N$ matrix-valued function $\mathbb{D}(z)=\left(D_{i j}(z)\right)_{1 \leq i, j \leq N}$ by

$$
D_{i j}(z)=m \delta_{i j}-\frac{e^{2}}{c^{2}} \frac{d-1}{d} \int_{\mathbb{R}^{d}} \frac{\hat{\rho}_{i}(k) \hat{\rho}_{j}(k)}{z-|k|^{2}} d k, \quad z \in \mathbb{C} \backslash[0, \infty)
$$

Lemma 2.4 Let (, ) denote the Euclidean inner product. Suppose $\vec{\rho} \in \tilde{P}$. Then the followings hold:

(1) The functions $D_{i j}(z, \kappa), 1 \leq i, j \leq N, \kappa>0$ are analytic in $\mathbb{C} \backslash[0, \infty)$.

(2) For $s \in[0, \infty)$ and $\kappa>0$, the pointwise limit $D_{ \pm i j}(s, \kappa) \equiv \lim _{h \rightarrow 0} D_{i j}(s \pm i h, \kappa)$ exists and has the following form

$$
\begin{aligned}
D_{ \pm i j}(s, \kappa) & =\frac{m}{\kappa^{2}} \delta_{i j}-\frac{1}{\kappa^{3}}\left(\frac{e^{2}}{c^{2}} \frac{\mathbf{V}_{d}}{2} \frac{d-1}{d}\right) H_{i j}(s) \pm \frac{2 \pi i}{\kappa^{3}}\left(\frac{e^{2}}{c^{2}} \frac{\mathbf{V}_{d}}{2} \frac{d-1}{d}\right) K_{i j}(s) \\
K_{i j}(s) & =\hat{\rho}_{i}(\sqrt{s}) \hat{\rho}_{j}(\sqrt{s}) s^{\frac{d}{2}-1} \\
H_{i j}(s) & =\lim _{\epsilon \rightarrow 0+} \int_{|s-x|>\epsilon} \frac{K_{i j}(x)}{s-x} d x
\end{aligned}
$$

where $\mathbf{V}_{d}=2 \pi^{\frac{d}{2}} / \Gamma\left(\frac{d}{2}\right)(\Gamma(z)$ is the gamma function). The convergence is uniform in $s \in[0, \infty)$; for any $\delta>0$, there exists $h_{0}>0$ independent of $s, \kappa$, such that for $0<{ }^{\forall} h \leq h_{0}$,

$$
\left|D_{i j}(s \pm i h, \kappa)-D_{ \pm i j}(s, \kappa)\right| \leq \frac{\delta}{\kappa^{3}}
$$


Moreover $H_{i j}(s)$ is Lipschitz continuous in $s \in[0, \infty)$ with the same order as that of $K_{i j}$ and contained in $L^{\epsilon}\left(\mathbb{R}^{d}\right)$ with some $\epsilon \geq 1$.

(3) Let $\kappa>0$ be sufficiently large. Put $\mathbb{D}_{ \pm}(s, \kappa)=\left(D_{ \pm i j}(s, \kappa)\right)_{1 \leq i, j \leq N}$. Then there exists a positive constant $d_{1}(\kappa)$ such that for $\left(w_{1}, \ldots, w_{N}\right)=\vec{w} \in \mathbb{C}^{N}$,

$$
\inf _{s \in[0, \infty)}\left|\left(\mathbb{D}_{ \pm}(s, \kappa) \vec{w}, \vec{w}\right)\right|>d_{1}(\kappa)|\vec{w}|^{2}
$$

(4) Let $\kappa>0$ be sufficiently large. Then there exists a positive constant $d_{2}(\kappa)$ such that for $\vec{w} \in \mathbb{C}^{N}$,

$$
\inf _{z \in \mathbb{C} \backslash[0, \infty)}|(\mathbb{D}(z, \kappa) \vec{w}, \vec{w})|>d_{2}(\kappa)|\vec{w}|^{2}
$$

Proof: The statements (1) and (2) are fundamental facts([9]). We shall prove (3). From (2) it follows that

$$
\left(\mathbb{D}_{ \pm}(s, \kappa) \vec{w}, \vec{w}\right)=\frac{m}{\kappa^{2}}\left\{|\vec{w}|^{2}-\frac{1}{\kappa} \frac{\lambda}{m}(H(s) \vec{w}, \vec{w})\right\} \pm 2 \pi i \frac{\lambda}{\kappa^{3}}(K(s) \vec{w}, \vec{w})
$$

where $\lambda=\frac{e^{2}}{c^{2}} \frac{\mathbf{V}_{d}}{2} \frac{d-1}{d}, H(s)=\left(H_{i j}(s)\right)_{1 \leq i, j \leq d}, K(s)=\left(K_{i j}(s)\right)_{1 \leq i, j \leq d}$. Since $H_{i j}$ is a Lipschitz continuous function and contained in $L^{\epsilon}([0, \infty))$, it is bounded. Hence we have

$$
|(H(s) \vec{w}, \vec{w})| \leq N \times \sup _{s \in[0, \infty), 1 \leq i, j \leq d}\left|H_{i j}(s)\right| \cdot|\vec{w}|^{2} \equiv \alpha|\vec{w}|^{2}
$$

Thus we can see that for sufficiently large $\kappa>0$

$$
\left|\left(\mathbb{D}_{ \pm}(s, \kappa) \vec{w}, \vec{w}\right)\right| \geq \frac{m}{\kappa^{2}}\left(1-\frac{1}{\kappa} \frac{\lambda}{m} \alpha\right)|\vec{w}|^{2} .
$$

Hence we get (3). We shall prove (4). From (2) it follows that for any $\eta>0$, there exists $\epsilon_{0}>0$ independent of $s \in[0, \infty)$ and $\kappa>0$ such that for $0<^{\forall} \epsilon \leq \epsilon_{0}$,

$$
\left|\left(\mathbb{D}_{ \pm}(s, \kappa) \vec{w}, \vec{w}\right)\right|-\frac{\eta}{\kappa^{3}}|\vec{w}|^{2} \leq|(\mathbb{D}(s \pm i \epsilon, \kappa) \vec{w}, \vec{w})|
$$


Hence we have

$$
|(\mathbb{D}(s \pm i \epsilon, \kappa) \vec{w}, \vec{w})| \geq\left\{\frac{m}{\kappa^{2}}\left(1-\frac{1}{\kappa} \frac{\lambda}{m} \alpha\right)-\frac{\eta}{\kappa^{3}}\right\}|\vec{w}|^{2}
$$

On the other hand, put $\Pi_{\epsilon_{0}}=\mathbb{C} \backslash\left\{x+i y|x \geq 0| y \mid, \leq \epsilon_{0}\right\}$. Then we see that for $x+i y \in \Pi_{\epsilon_{0}}$

$$
\begin{aligned}
(\mathbb{D}(x+i y, \kappa) \vec{w}, \vec{w})= & \frac{m}{\kappa^{2}}\left(|\vec{w}|^{2}-\frac{1}{\kappa} \frac{\lambda}{m} \int_{0}^{\infty} \frac{(x-s)\left|\sum_{i=1}^{N} w_{i} \hat{\rho}_{i}(\sqrt{s})\right|^{2} s^{\frac{d}{2}-1}}{(x-s)^{2}+y^{2}} d s\right) \\
& +i \frac{\lambda}{\kappa^{3}} \int_{0}^{\infty} \frac{y\left|\sum_{i=1}^{N} w_{i} \hat{\rho}(\sqrt{s})\right|^{2} s^{\frac{d}{2}-1}}{(x-s)^{2}+y^{2}} d s .
\end{aligned}
$$

Noting that $|a b| / a^{2}+b^{2} \leq 1 / 2$, we have

$$
\begin{aligned}
\left|\int_{0}^{\infty} \frac{(x-s)\left|\sum_{i=1}^{N} w_{i} \hat{\rho}_{i}(\sqrt{s})\right|^{2} s^{\frac{d}{2}-1}}{(x-s)^{2}+y^{2}} d s\right| & \leq \frac{1}{2|y|} \int_{0}^{\infty}\left|\sum_{i=1}^{N} w_{i} \hat{\rho}_{i}(\sqrt{s})\right|^{2} s^{\frac{d}{2}-1} d s \\
& \leq \frac{1}{2 \epsilon_{0}} \int_{0}^{\infty} \sum_{i=1}^{N}\left|\hat{\rho}_{i}(\sqrt{s})\right|^{2} s^{\frac{d}{2}-1} d s|\vec{w}|^{2} \\
& \equiv \frac{\beta}{\epsilon_{0}}|\vec{w}|^{2}
\end{aligned}
$$

Since $\epsilon_{0}$ is independent of $\kappa>0$, we see that for sufficiently large $\kappa>0$,

$$
|(\mathbb{D}(x+i y, \kappa) \vec{w}, \vec{w})| \geq \frac{m}{\kappa^{2}}\left(1-\frac{1}{\kappa} \frac{\lambda}{m} \frac{\beta}{\epsilon_{0}}\right)|\vec{w}|^{2}, \quad x+i y \in \Pi_{\epsilon_{0}} .
$$

Combining (2.3) and (2.4), we get (4).

From Lemma 2.4 (3) and (4) it follows that for sufficiently large $\kappa>0$, there exist the inverse matrices to $\mathbb{D}(z, \kappa)$ and $\mathbb{D}_{ \pm}(s, \kappa)$, which satisfy

$$
\begin{aligned}
& \sup _{s \in[0, \infty)}\left|\left(\mathbb{D}_{ \pm}^{-1}(s, \kappa) \vec{w}_{1}, \vec{w}_{2}\right)\right|<\frac{1}{d_{1}(\kappa)}\left|\vec{w}_{1}\right|\left|\vec{w}_{2}\right| \\
& \sup _{z \in \mathbb{C} \backslash[0, \infty)}\left|\left(\mathbb{D}^{-1}(z, \kappa) \vec{w}_{1}, \vec{w}_{2}\right)\right|<\frac{1}{d_{2}(\kappa)}\left|\vec{w}_{1}\right|\left|\vec{w}_{2}\right| .
\end{aligned}
$$

We set for $\vec{\rho} \in \tilde{P}$ and sufficiently large $\kappa>0$

$$
Q(k, \kappa) \equiv \mathbb{D}_{+}^{-1}\left(k^{2}, \kappa\right)\left(\begin{array}{c}
\hat{\rho}_{1}(k) \\
\vdots \\
\hat{\rho}_{N}(k)
\end{array}\right) \equiv\left(Q_{1}(k, \kappa), \ldots, Q_{N}(k, \kappa)\right)
$$


For later use in Appendix, we note that for all $s \in[0, \infty)$

$$
D_{+i j}(s, \kappa)-D_{-i j}(s, \kappa)=2 \pi i \frac{1}{\kappa^{3}}\left(\frac{e^{2}}{c^{2}} \mathbf{V}_{d} \frac{d-1}{d}\right) \hat{\rho}_{i}(\sqrt{s}) \hat{\rho}_{j}(\sqrt{s}) s^{\frac{d}{2}-1}
$$

Put $\mathbb{D}^{-1}(z)=\left(D_{i j}^{-1}(z)\right)_{1 \leq i j \leq N}, \mathbb{D}_{ \pm}^{-1}(s)=\left(D_{ \pm i j}^{-1}(s)\right)_{1 \leq i j \leq N}$. Then (2.7) implies that

$$
\frac{2 \pi i}{\kappa^{3}}\left(\frac{e^{2}}{c^{2}} \mathbf{V}_{d} \frac{d-1}{d}\right) \sum_{k, l=1}^{N} D_{-i k}^{-1}(s, \kappa) D_{+j l}^{-1}(s, \kappa) \hat{\rho}_{k}(\sqrt{s}) \hat{\rho}_{l}(\sqrt{s}) s^{\frac{d}{2}-1}=D_{-i j}(s, \kappa)-D_{+j i}(s, \kappa)
$$

\section{Remark 2.5}

(1) In $[3,4,6]$, the authors define functions $D_{ \pm}(s)$ corresponding to $\mathbb{D}_{ \pm}(s)$ defined in this paper. The function $1 / D_{ \pm}(s, \kappa)$ can be well defined for some $\rho$ and any $\kappa>0$. However, in our case, we do not know whether $\mathbb{D}_{ \pm}(s, \kappa)$ has the inverse or not for all $\kappa>0$. But since, in this paper, we focus on an asymptotic behavior as $\kappa \rightarrow \infty$, it is sufficient to consider the case where $\kappa$ is sufficiently large.

(2) For the proof of Lemma 2.4, we do not need Definition 2.1 (4).

We define operators $G_{h}(h>0)$ by

$$
\left(G_{h} f\right)(k)=\int_{\mathbb{R}^{d}} \frac{f\left(k^{\prime}\right)}{\left(k^{2}-k^{\prime 2}+i h\right)\left(k k^{\prime}\right)^{\frac{d}{2}-1}} d k^{\prime}
$$

It is well known and not so hard to see that $G_{h}$ are bounded linear operators on $L^{2}\left(\mathbb{R}^{d}\right)$ and the strong $\operatorname{limits}_{\lim _{h \rightarrow 0}} G_{h} \equiv G$ exists ([4]). Furthermore $G$ is skew symmetric $\left(G^{*}=-G\right)$. For sufficiently large $\kappa>0$, we can define the following operators:

$$
T_{\mu \nu}(\kappa) f \equiv \delta_{\mu \nu} f+\frac{1}{\kappa^{3}} \frac{e^{2}}{c^{2}} \sum_{j=1}^{N} Q_{j}(\kappa) \omega^{\frac{d}{2}-1} G \omega^{\frac{d}{2}-1} d_{\mu \nu} \hat{\rho}_{j} f, \quad 1 \leq \mu, \nu \leq d
$$

Lemma 2.6 Suppose that $\vec{\rho} \in \widetilde{P}$ and $\kappa>0$ is sufficiently large. Then the following holds.

(1) $T_{\mu \nu}(\kappa)$ and $T_{\mu \nu}^{*}(\kappa)$ are bounded operators on $M_{\alpha}, \alpha=-1,0,1$ and $\left(T_{\mu \nu}(\kappa) f\right)=T_{\mu \nu}(\kappa) \tilde{f}$. 
(2) Put $D_{i j}^{-1}(0, \kappa) \equiv D_{ \pm i j}^{-1}(0, \kappa)$ and let $f \in M_{-1}$. Then

$$
\left\langle d_{\nu \alpha} \frac{Q_{i}(\kappa)}{\sqrt{\omega^{3}}}, \frac{1}{\sqrt{\omega}} T_{\mu \nu}(\kappa) f\right\rangle_{L^{2}\left(\mathbb{R}^{d}\right)}=\left\langle d_{\mu \alpha} \sum_{j=1}^{N} D_{i j}^{-1}(0, \kappa) \frac{\hat{\rho}_{j}}{\sqrt{\omega^{3}}}, \frac{f}{\sqrt{\omega}}\right\rangle_{L^{2}\left(\mathbb{R}^{d}\right)}, i=1, \ldots, N
$$

(3) $\left[\omega^{2}, T_{\mu \nu}^{*}(\kappa)\right]=-\sum_{i=1}^{N} \frac{1}{\kappa^{3}} \frac{e^{2}}{c^{2}}\left\langle Q_{i}(\kappa), \cdot\right\rangle_{L^{2}\left(\mathbb{R}^{d}\right)} d_{\mu \nu} \hat{\rho}_{i}$.

(4) $T_{\mu \nu}(\kappa) \hat{\rho}_{j}=\delta_{\mu \nu} \frac{m}{\kappa^{2}} Q_{j}(\kappa)$.

(5) $T_{\mu \nu}^{*}(\kappa) d_{\nu \alpha} T_{\alpha \beta}(\kappa)=d_{\mu \beta}$.

(6) $e_{\mu}^{r} T_{\mu \nu}(\kappa) d_{\nu \alpha} T_{\alpha \beta}(\kappa) e_{\beta}^{s}=\delta_{r s}$.

Proof: See Appendix.

In the rest of this section, we fix sufficiently large $\kappa>0$ and omit $\kappa$ in notations for simplicity. Define $\widehat{A}_{\mu}(f)=A_{\mu}(\hat{f})$ and $\widehat{\Pi}_{\mu}(f)=\Pi_{\mu}(\hat{f})$. We put

$$
\begin{aligned}
B^{(r)}(f, p)= & \frac{1}{\sqrt{2}}\left\{\widehat{A}_{\mu}\left(\frac{1}{\sqrt{\hbar}} T_{\mu \nu}^{*} e_{\nu}^{r} \sqrt{c \omega} f\right)+i \widehat{\Pi}_{\mu}\left(\frac{1}{\sqrt{\hbar}} T_{\mu \nu}^{*} e_{\nu}^{r} \frac{f}{\sqrt{c \omega}}\right)\right. \\
& \left.+\sum_{j=1}^{N} \hbar p_{\nu}^{j}\left\langle\frac{e}{\sqrt{\hbar}} \frac{Q_{j} e_{\nu}^{r}}{(c \omega)^{\frac{3}{2}}}, f\right\rangle_{L^{2}\left(\mathbb{R}^{d}\right)}\right\}, \quad f \in M_{0} \\
B^{\dagger(r)}(f, p)= & \frac{1}{\sqrt{2}}\left\{\widehat{A}_{\mu}\left(\frac{1}{\sqrt{\hbar}} \bar{T}_{\mu \nu}^{*} \tilde{e}_{\nu}^{r} \sqrt{c \omega} \tilde{f}\right)-i \widehat{\Pi}_{\mu}\left(\frac{1}{\sqrt{\hbar}} \bar{T}_{\mu \nu}^{*} \tilde{e}_{\nu}^{r} \frac{\tilde{f}}{\sqrt{c \omega}}\right)\right. \\
& \left.+\sum_{j=1}^{N} \hbar p_{\nu}^{j}\left\langle\frac{e}{\sqrt{\hbar}} \frac{\bar{Q}_{j} e_{\nu}^{r}}{(c \omega)^{\frac{3}{2}}}, f\right\rangle_{L^{2}\left(\mathbb{R}^{d}\right)}\right\}, f \in M_{0}, p=\left(p^{1}, \ldots, p^{N}\right) \in \mathbb{R}^{d N}
\end{aligned}
$$

By the definition of $A_{\mu}(f)$ and $\Pi_{\mu}(f)$, for the vector of the form $\mathbf{f}=f_{1} \oplus \ldots \oplus f_{d-1} \in \mathcal{W}$, we see that for $p=\left(p^{1}, \ldots, p^{N}\right) \in \mathbb{R}^{d N}$

$$
\begin{aligned}
& B(\mathbf{f}, p) \equiv \sum_{r=1}^{d-1} B^{(r)}\left(f_{r}, p\right)=a^{\dagger}\left(\mathbf{W}_{-} \mathbf{f}\right)+a\left(\mathbf{W}_{+} \mathbf{f}\right)+\sum_{j=1}^{N}\left\langle\mathbf{L}_{j} p^{j}, \mathbf{f}\right\rangle_{L^{2}\left(\mathbb{R}^{d}\right)} \\
& B^{\dagger}(\mathbf{f}, p) \equiv \sum_{r=1}^{d-1} B^{\dagger(r)}\left(f_{r}, p\right)=a^{\dagger}\left(\overline{\mathbf{W}}_{+} \mathbf{f}\right)+a\left(\overline{\mathbf{W}}_{-} \mathbf{f}\right)+\sum_{j=1}^{N}\left\langle\overline{\mathbf{L}}_{j} p^{j}, \mathbf{f}\right\rangle_{L^{2}\left(\mathbb{R}^{d}\right)}
\end{aligned}
$$


where

$$
\begin{aligned}
\mathbf{W}_{ \pm} & =\left(W_{ \pm}^{(\tau, s)}\right)_{1 \leq r, s \leq d-1} \\
\mathbf{L}_{j} & =\left(L_{\mu j}^{r}\right)_{1 \leq \mu \leq d, 1 \leq r \leq d-1}=\left(\begin{array}{lll}
L_{1 j}^{1} & \cdots & L_{d j}^{1} \\
\vdots & & \vdots \\
L_{1 j}^{d-1} & \cdots & L_{d j}^{d-1}
\end{array}\right), j=1, \ldots, N
\end{aligned}
$$

where

$$
\begin{aligned}
W_{+}^{(r, s)} f & =\frac{1}{2}\left(\frac{1}{\sqrt{\omega}} e_{\mu}^{r} T_{\mu \nu}^{*} e_{\nu}^{s} \sqrt{\omega}+\sqrt{\omega} e_{\mu}^{r} T_{\mu \nu}^{*} e_{\nu}^{s} \frac{1}{\sqrt{\omega}}\right) f \\
W_{-}^{(r, s)} f & =\frac{1}{2}\left(\frac{1}{\sqrt{\omega}} e_{\mu}^{r} T_{\mu \nu}^{*} \tilde{e}_{\nu}^{s} \sqrt{\omega}-\sqrt{\omega} e_{\mu}^{r} T_{\mu \nu}^{*} \tilde{e}_{\nu}^{s} \frac{1}{\sqrt{\omega}}\right) \tilde{f} \\
L_{\mu j}^{r} & =\frac{e \sqrt{\hbar} e_{\mu}^{r} Q_{j}}{\sqrt{2 c^{3} \omega^{3}}} .
\end{aligned}
$$

We see that, by Lemma $2.6(1), \mathbf{W}_{ \pm}$is a bounded operator on $\mathcal{W}$. By virtue of Lemma 2.6 (5) and (6), one can easily see that $\mathbf{W}_{ \pm}$satisfy the following algebraic relations:

$$
\begin{aligned}
& \mathbf{W}_{+}^{*} \mathbf{W}_{+}-\mathbf{W}_{-}^{*} \mathbf{W}_{-}=I, \\
& \overline{\mathbf{W}}_{+}^{*} \mathbf{W}_{-}-\overline{\mathbf{W}}_{-}^{*} \mathbf{W}_{+}=0, \\
& \mathbf{W}_{+} \mathbf{W}_{+}^{*}-\overline{\mathbf{W}}_{-} \overline{\mathbf{W}}_{-}^{*}=I, \\
& \mathbf{W}_{-} \mathbf{W}_{+}^{*}-\overline{\mathbf{W}}_{+} \overline{\mathbf{W}}_{-}^{*}=0 .
\end{aligned}
$$

We put $\mathcal{W}_{\alpha}=\underbrace{M_{\alpha} \oplus \ldots \oplus M_{\alpha}}_{d-1}, \alpha \in \mathbb{R}$. These relations $(2.10)$ imply that on $\mathcal{F}^{\infty}(\mathcal{W})$ for $\mathbf{f}, \mathbf{g} \in \mathcal{W}_{0}$

$$
\begin{aligned}
{\left[B(\mathbf{f}, p), B^{\dagger}(\mathbf{g}, p)\right] } & =\langle\mathbf{f}, \mathbf{g}\rangle_{\mathcal{W}}, \\
{\left[B^{\sharp}(\mathbf{f}, p), B^{\sharp}(\mathbf{g}, p)\right] } & =0,
\end{aligned}
$$

and for $\Phi, \Psi \in \mathcal{F}^{\infty}(\mathcal{W})$

$$
\left\langle B^{\dagger}(\mathbf{f}, p) \Phi, \Psi\right\rangle_{\mathcal{F}(\mathcal{W})}=\langle\Phi, B(\overline{\mathbf{f}}, p) \Psi\rangle_{\mathcal{F}(\mathcal{W})}
$$


Lemma 2.7 For $\mathbf{f} \in \mathcal{W}_{0} \cap \mathcal{W}_{2}, p \in \mathbb{R}^{d N}$ and $\vec{\rho} \in \tilde{P}$, we have

$$
\left[H_{\bar{\rho}}(p), B^{\sharp}(\mathbf{f}, p)\right]= \pm B^{\sharp}(\hbar c \tilde{\omega} \mathbf{f}, p) \text {, on } \mathcal{F}^{\infty}(\mathcal{W}) \cap D\left(H_{b}^{\frac{3}{2}}\right) \text {, }
$$

where $+\left(\right.$ resp.-) corresponds to $B^{\dagger}$ (resp.B).

Proof: Suppose that $\mathbf{f} \in \mathcal{W}_{-2} \cap \mathcal{W}_{0} \cap \mathcal{W}_{2}$. Then by Lemma 2.6 (3) and (4), one can directly see that (2.11) holds. Next by a limiting argument, one can get (2.11) for $\mathbf{f} \in \mathcal{W}_{0} \cap \mathcal{W}_{2}$.

Define

$$
\begin{aligned}
A(\mathbf{f}, p) & \equiv \frac{1}{\sqrt{2}}\left(B^{\dagger}(\mathbf{f}, p)+B(\overline{\mathbf{f}}, p)\right), \\
\Pi(\mathbf{f}, p) & \equiv \frac{i}{\sqrt{2}}\left(B^{\dagger}(\mathbf{f}, p)-B(\overline{\mathbf{f}}, p)\right), \quad \mathbf{f} \in \mathcal{W}_{0}
\end{aligned}
$$

We can easily see that the operators $\left.A(\mathbf{f}, p)\right|_{\mathcal{F} \infty(\mathcal{W})}$ and $\left.\Pi(\mathbf{f}, p)\right|_{\mathcal{F} \infty(\mathcal{W})}$ are essentially selfadjoint by the Nelson analytic vector theorem [10,Theorem X.39]. We denote the self-adjoint extensions by the same symbols.

Theorem 2.8 Suppose $\vec{\rho} \in \widetilde{P}$. Then for $\mathbf{f} \in \mathcal{W}_{0}$

$$
\begin{aligned}
& \exp \left(i \frac{t}{\hbar} H_{\vec{\rho}}(p)\right) A(\mathbf{f}, p) \exp \left(-i \frac{t}{\hbar} H_{\vec{\rho}}(p)\right)=A\left(e^{i c \tilde{\omega} t} \mathbf{f}, p\right) \\
& \exp \left(i \frac{t}{\hbar} H_{\vec{\rho}}(p)\right) \Pi(\mathbf{f}, p) \exp \left(-i \frac{t}{\hbar} H_{\vec{\rho}}(p)\right)=\Pi\left(e^{i c \tilde{\omega} t} \mathbf{f}, p\right) .
\end{aligned}
$$

Proof: We only show an outline of the proof. For simplicity, put $A\left(e^{i c \tilde{\omega} t} \mathbf{f}, p\right)=A(\mathbf{f}, p, t)$. Let $C^{\infty}\left(H_{b}\right)=\cap_{n=1}^{\infty} D\left(H_{b}^{n}\right)$. We can easily see that, by Lemma $2.7,\left\langle e^{i A(\mathbf{f}, p, t)} \Psi, \Phi\right\rangle, \Psi, \Phi \in$ $C^{\infty}\left(H_{b}\right) \cap \mathcal{F}^{\infty}(\mathcal{W}), \mathbf{f} \in \mathcal{W}_{-2} \cap \mathcal{W}_{0} \cap \mathcal{W}_{2}$, is differentiable in $t$ with

$$
\frac{d}{d t}\left\langle e^{i A(\mathbf{f}, p, t)} \Psi, \Phi\right\rangle_{\mathcal{F}(\mathcal{W})}=\left\langle\frac{i}{\hbar} e^{i A(\mathbf{f}, p, t)} \Psi, H_{\vec{\rho}}(p) \Phi\right\rangle_{\mathcal{F}(\mathcal{W})}-\left\langle\frac{i}{\hbar} H_{\vec{\rho}}(p) \Psi, e^{-i A(\mathbf{f}, p, t)} \Phi\right\rangle_{\mathcal{F}(\mathcal{W})}
$$

From (2.14) it follows that

$$
\frac{d}{d t}\left\langle e^{-i \frac{t}{\hbar} H_{\vec{\rho}}(p)} e^{i A(\mathbf{f}, p, t)} e^{i \frac{t}{\hbar} H_{\vec{\rho}}(p)} \Psi, \Phi\right\rangle_{\mathcal{F}(\mathcal{W})}=0, \quad \Psi, \Phi \in D\left(H_{b}\right), \mathbf{f} \in \mathcal{W}_{0}
$$


Hence

$$
e^{i s A(\mathbf{f}, p, 0)}=e^{i \frac{t}{\hbar} H_{\vec{\rho}}(p)} e^{i s A(\mathbf{f}, p, t)} e^{-i \frac{t}{\hbar} H_{\vec{\rho}}(p)}, \quad \text { on } C^{\infty}\left(H_{b}\right) \cap \mathcal{F}^{\infty}(\mathcal{W})
$$

By a limiting argument, one can see that (2.15) holds for $\Phi, \Psi \in D\left(H_{b}\right), \mathbf{f} \in \mathcal{W}_{0}$. Since the both sides of (2.15) are one parameter unitary groups in $s \in \mathbb{R}$, Stone's theorem yields (2.12). (2.13) is quite similar to (2.12). Thus we get the desired result.

For $t \in \mathbb{R}$, we define operators in $\mathcal{F}(\mathcal{W})$ by

$$
\begin{aligned}
A_{\mu}(f, t \mid p)= & \frac{1}{\sqrt{2}} \sum_{r=1}^{d-1}\left\{B^{\dagger(r)}\left(\frac{\sqrt{\hbar}}{\sqrt{c \omega}} e^{i c \omega t} e_{\nu}^{r} \bar{T}_{\mu \nu} \hat{f}, p\right)+B^{(r)}\left(\frac{\sqrt{\hbar}}{\sqrt{c \omega}} e^{-i \omega \omega t} e_{\nu}^{r} T_{\mu \nu} \tilde{\hat{f}}, p\right)\right\} \\
& -e \sum_{i, j=1}^{N} \hbar p_{\nu}^{i}\left\langle d_{\mu \nu} D_{i j}^{-1}(0) \frac{\hat{\rho}_{j}}{\sqrt{(c \omega)^{3}}}, \frac{\hat{f}}{\sqrt{c \omega}}\right\rangle_{L^{2}\left(\mathbb{R}^{d}\right)}, \quad \hat{f} \in M_{-1}, \mu=1, \ldots, d .
\end{aligned}
$$

Form Lemma 2.5 (2) (5) and (6) it follows that

$$
A_{\mu}(f, 0 \mid p)=A_{\mu}(f)
$$

Corollary 2.9 Suppose $\vec{\rho} \in \widetilde{P}$. Then the operator $A_{\mu}(f, t \mid p)$ is the Heisenberg field with

$$
\exp \left(i \frac{t}{\hbar} H_{\vec{\rho}}(p)\right) A_{\mu}(f) \exp \left(-i \frac{t}{\hbar} H_{\vec{\rho}}(p)\right)=A_{\mu}(f, t \mid p)
$$

Proof: It is enough to show (2.17) for a real-valued function $f$. For a real-valued function $f$ such that $\hat{f} \in M_{-1}$, we can see that

$$
\begin{aligned}
A_{\mu}(f)= & \frac{1}{\sqrt{2}} \sum_{r=1}^{d-1}\left\{B^{\dagger(r)}\left(\frac{\sqrt{\hbar}}{\sqrt{c \omega}} e_{\nu}^{r} \bar{T}_{\mu \nu} \hat{f}, p\right)+B^{(r)}\left(\frac{\sqrt{\hbar}}{\sqrt{c \omega}} e_{\nu}^{r} \bar{T}_{\mu \nu} \hat{f}, p\right)\right\} \\
& -e \sum_{i, j=1}^{N} \hbar p_{\nu}^{i}\left\langle d_{\mu \nu} D_{i j}^{-1}(0) \frac{\hat{\rho}_{j}}{\sqrt{(c \omega)}}, \frac{\hat{f}}{\sqrt{c \omega}}\right\rangle .
\end{aligned}
$$

Hence (2.17) follows from Theorem 2.8.

Corollary 2.10 Suppose $\vec{\rho} \in \widetilde{P}$. Then for $\Phi \in D\left(H_{b}\right)$,

$$
\exp \left(i \frac{t}{\hbar} H_{\bar{\rho}}(p)\right) B^{\sharp}(\mathbf{f}, p) \exp \left(-i \frac{t}{\hbar} H_{\bar{\rho}}(p)\right) \Phi=B^{\sharp}\left(e^{i c \tilde{\omega} t} \mathbf{f}, p\right) \Phi
$$


Proof: Note that $D\left(B^{\sharp}(\mathbf{f}, p)\right) \subset D\left(H_{b}\right)=D\left(H_{\vec{\rho}}(p)\right)$. Thus from (2.12) and (2.13) it follows that on $D\left(H_{b}\right)$

$$
\begin{aligned}
e^{i \frac{t}{\hbar} H_{\vec{\rho}}(p)} A(\mathbf{f}, p) e^{-i \frac{t}{\hbar} H_{\vec{\rho}}(p)} & =\frac{1}{\sqrt{2}}\left\{e^{i \frac{t}{\hbar} H_{\vec{\rho}}(p)} B^{\dagger}(\mathbf{f}, p) e^{-i \frac{t}{\hbar} H_{\vec{\rho}}(p)}+e^{i \frac{t}{\hbar} H_{\vec{\rho}}(p)} B(\overline{\mathbf{f}}, p) e^{-i \frac{t}{\hbar} H_{\bar{\rho}}(p)}\right\}, \\
& =\frac{1}{\sqrt{2}}\left\{B^{\dagger}\left(e^{i c \tilde{\omega} t} \mathbf{f}\right)+B\left(e^{-i c \tilde{\omega} t \overline{\mathbf{f}}}\right)\right\} \\
e^{i \frac{t}{\hbar} H_{\vec{\rho}}(p)} \Pi(\mathbf{f}, p) e^{-i \frac{t}{\hbar} H_{\vec{\rho}}(p)} & =\frac{i}{\sqrt{2}}\left\{e^{i \frac{t}{\hbar} H_{\vec{\rho}}(p)} B^{\dagger}(\mathbf{f}, p) e^{\left(-i \frac{t}{\hbar} H_{\vec{\rho}}(p)\right.}-e^{i \frac{t}{\hbar} H_{\vec{\rho}}(p)} B(\overline{\mathbf{f}}, p) e^{-i \frac{t}{\hbar} H_{\vec{\rho}}(p)}\right\} \\
& =\frac{i}{\sqrt{2}}\left\{B^{\dagger}\left(e^{i c \tilde{\omega} t} \mathbf{f}\right)-B\left(e^{-i c \tilde{\omega} t \overline{\mathbf{f}}}\right)\right\}
\end{aligned}
$$

Thus the corollary follows.

\section{BOGOLIUBOV TRANSFORMATIONS AND SCAL- ING LIMITS}

In this section, we construct a unitary operator which implements a unitary equivalence of the Pauli-Fierz Hamiltonian and a decoupled Hamiltonian. Moreover we investigate a scaling limit of the Pauli-Fierz Hamiltonian. Unless otherwise stated in this section, we suppose that $\kappa>0$ is sufficiently large. Since the bounded operators $W_{-}^{r, s}(\kappa)$ have integral kernels

$$
W_{-}^{(r, s)}\left(k, k^{\prime}, \kappa\right)=\frac{1}{\kappa^{3}} \frac{e^{2}}{c^{2}} \frac{e_{\mu}^{r}(k) e_{\mu}^{s}\left(k^{\prime}\right) \sum_{j=1}^{N} \hat{\rho}_{j}(k) \bar{Q}_{j}\left(k^{\prime}, \kappa\right)}{2\left(|k|+\left|k^{\prime}\right|\right)\left(|k|\left|k^{\prime}\right|\right)^{\frac{1}{2}}}
$$

such that $W_{-}^{(r, s)}(\kappa) \in L^{2}\left(\mathbb{R}^{d} \times \mathbb{R}^{d}\right)$, the operator $\mathbf{W}_{-}(\kappa)$ is a Hilbert Schmidt operator on $\mathcal{W}$. Then from (2.9) and (2.10) it follows that there exist two unitary operators $U(\kappa)(p$ independent) and $S(p, \kappa)$ such that ([6,Section III])

$$
U^{-1}(\kappa) S(p, \kappa)^{-1} B^{\sharp}(\mathbf{f}, p, \kappa) S(p, \kappa) U(\kappa)=a^{\sharp}(\mathbf{f}), \quad \mathbf{f} \in \mathcal{W} .
$$

Concretely $S(p, \kappa)$ is given by

$$
S(p, \kappa)=\exp \left(\sum_{i, j=1}^{N} \frac{e \hbar}{\kappa^{2}} p_{\mu}^{i}\left\{a\left(\oplus_{r=1}^{d-1} \frac{e_{\mu}^{r} D_{i j}^{-1}(0, \kappa) \hat{\rho}_{j}}{\sqrt{2 \hbar c^{3} \omega^{3}}}\right)-a^{\dagger}\left(\oplus_{r=1}^{d-1} \frac{e_{\mu}^{r} D_{i j}^{-1}(0, \kappa) \hat{\rho}_{j}}{\sqrt{2 \hbar c^{3} \omega^{3}}}\right)\right\}\right)
$$


Theorem 3.1 Suppose $\vec{\rho} \in \tilde{P}$. Then putting $S(p, \kappa) U(\kappa)=\mathcal{U}(p, \kappa)$, we see that $\mathcal{U}(p, \kappa)$ maps $D\left(H_{b}\right)$ onto itself with

$$
\mathcal{U}(p, \kappa) H_{\vec{\rho}}(p, \kappa) \mathcal{U}^{-1}(p, \kappa)=\kappa H_{b}+E(p, \kappa)
$$

where

$$
\begin{aligned}
E(p, \kappa) & =\frac{\hbar^{2}}{2 m} \sum_{i=1}^{N} \sum_{\mu=1}^{d}\left(\kappa p_{\mu}^{i}+\kappa \tilde{p}_{\mu}^{i}(\kappa)\right)^{2}+\square(\kappa), \\
\widetilde{p}_{\mu}^{i}(\kappa) & =\sum_{j=1}^{N} p_{\nu}^{j} \Delta_{\nu \mu}^{j i}(\kappa) \\
\Delta_{\nu \mu}^{j i}(\kappa) & =\frac{1}{\kappa^{3}} \frac{e^{2}}{2 c^{2}} \sum_{k=1}^{N} \sum_{r, s=1}^{d-1}\left\langle\frac{e_{\nu}^{r} D_{j k}^{-1}(0, \kappa) \hat{\rho}_{k}}{\sqrt{\omega^{3}}},\left(I+\mathbf{W}_{-}(\kappa) \mathbf{W}_{+}^{-1}(\kappa)\right)^{(r, s)} \frac{e_{\mu}^{s} \hat{\rho}_{i}}{\sqrt{\omega}}\right\rangle_{L^{2}\left(\mathbb{R}^{d}\right)}, \\
\square(\kappa) & =\frac{e^{2} \hbar}{4 m c} \sum_{i=1}^{N} \sum_{r, s=1}^{d-1}\left\langle\frac{e_{\mu}^{r} \hat{\rho}_{i}}{\sqrt{\omega}},\left(I-\mathbf{W}_{-}(\kappa) \mathbf{W}_{+}^{-1}(\kappa)\right)^{(r, s)} \frac{e_{\mu}^{s} \hat{\rho}_{i}}{\sqrt{\omega}}\right\rangle_{L^{2}\left(\mathbb{R}^{d}\right)} .
\end{aligned}
$$

Proof: For simplicity, we omit the symbol $\kappa$. Put $\mathcal{U}(p) \Omega \equiv \Omega(p)$. From [6,Proposition 2.4, Lemma 5.9] it follows that $\Omega(p) \in D\left(H_{b}\right)$. Then $\Omega(p) \in D(B(\mathbf{f}, p))$. By virtue of Corollary 2.10 and (3.1), we can see that for all $\mathbf{f} \in \mathcal{W}$

$$
B(\mathbf{f}, p) \exp \left(i \frac{t}{\hbar} H_{\vec{\rho}}(p)\right) \Omega(p)=0
$$

The equation (3.3) implies that there exists a positive constant $E(p)$ such that

$$
\exp \left(i \frac{t}{\hbar} H_{\vec{\rho}}(p)\right) \Omega(p)=\exp \left(i \frac{t}{\hbar} E(p)\right) \Omega(p)
$$

Hence from Corollary 2.10, (3.1), (3.4) and the denseness of

$$
\mathcal{L}\left\{B^{\dagger}\left(\mathbf{f}_{1}\right) \ldots B^{\dagger}\left(\mathbf{f}_{n}\right) \Omega(p), \Omega(p) \mid \mathbf{f}_{j} \in \mathcal{W}, j=1, \ldots, n, n \geq 1\right\}
$$

one can get (3.2)(we refer to [6,Lemma 5.12]). Noting that ([6,Lemma 2.2])

$$
a\left(\oplus_{r=1}^{d-1} \frac{\sqrt{\hbar} e_{\mu}^{r} \hat{\rho}_{i}}{\sqrt{2 c \omega}}\right) \Omega(p)=\left\{-\tilde{p}_{\mu}^{i}-a^{\dagger}\left(\oplus_{r=1}^{d-1} \sum_{s=1}^{d-1}\left(\mathbf{W}_{-} \mathbf{W}_{+}^{-1}\right)^{(r, s)} \frac{\sqrt{\hbar} e_{\mu}^{s} \hat{\rho}_{i}}{\sqrt{2 c \omega}}\right)\right\} \Omega(p)
$$


one can easily get $E(p)$ by

$$
E(p)=\frac{<H_{\vec{\rho}}(p) \Omega(p), \Omega>_{\mathcal{F}(\mathcal{W})}}{<\Omega(p), \Omega>_{\mathcal{F}(\mathcal{W})}}
$$

This completes the proof.

The positive constant $E(p, \kappa)$ can be rewritten as

$$
E(p, \kappa)=\frac{\kappa^{2} \hbar^{2}}{2 m} p^{2}+E^{R E N}(p, \kappa)+\tilde{E}(p, \kappa)
$$

where

$$
\begin{aligned}
\tilde{E}(p, \kappa) & =\frac{\kappa^{2} \hbar^{2}}{2 m} \sum_{i, j=1}^{N} \sum_{\mu, \nu=1}^{d} p_{\mu}^{i} b_{\mu \nu}^{i j}(\kappa) p_{\nu}^{j} \\
b_{\mu \nu}^{i j}(\kappa) & =\sum_{k=1}^{N} \sum_{\alpha=1}^{d}\left(\frac{\Delta_{\nu \alpha}^{j k}(\kappa)+\overline{\Delta_{\nu \alpha}^{j k}}(\kappa)}{2}\right)\left(\frac{\Delta_{\mu \alpha}^{i k}(\kappa)+\overline{\Delta_{\mu \alpha}^{i k}}(\kappa)}{2}\right), \\
E^{R E N}(p, \kappa) & =E(p, \kappa)-\frac{\kappa^{2} \hbar^{2}}{2 m} p^{2}-\tilde{E}(p, \kappa) .
\end{aligned}
$$

Let $M(K)$ be the set of $K \times K$ complex matrices. Note that since $\left(b_{\mu \nu}^{i j}(\kappa)\right)_{1 \leq i, j \leq N, 1 \leq \mu, \nu \leq d} \in$ $M(N) \otimes M(d) \cong M(d N)$ is nonnegative and symmetric, we have $\tilde{E}(p, \kappa) \geq 0$ for $p \in \mathbb{R}^{d N}$. We define $H_{\vec{\rho}}{ }^{R E N}(p, \kappa)$ and $\widetilde{H_{\vec{\rho}}}(p, \kappa)$ by

$$
\begin{aligned}
H_{\vec{\rho}}^{R E N}(p, \kappa) & =-E^{R E N}(p, \kappa)+\kappa H_{b}+\frac{1}{2 m} \sum_{j=1}^{N} \sum_{\mu=1}^{d}\left(-2 \kappa e \hbar p_{\mu}^{j} A_{\mu}\left(\rho_{j}\right)+e^{2} A_{\mu}\left(\rho_{j}\right)^{2}\right), \\
\widetilde{H_{\vec{\rho}}}(p, \kappa) & =\widetilde{E}(p, \kappa)+\kappa H_{b} .
\end{aligned}
$$

Then one can see that

$$
\begin{aligned}
H_{\vec{\rho}}^{R E N}(\kappa) \equiv & \mathbf{F}^{-1}\left(\int_{\mathbb{R}^{d N}}^{\oplus} H_{\vec{\rho}}^{R E N}(p, \kappa) d p\right) \mathbf{F} \\
= & -E^{R E N}(D, \kappa) \otimes I+\kappa I \otimes H_{b} \\
& +\frac{1}{2 m} \sum_{j=1}^{N} \sum_{\mu=1}^{d}\left(-2 \kappa e \hbar i D_{\mu}^{j} \otimes A_{\mu}\left(\rho_{j}\right)+e^{2} I \otimes A_{\mu}\left(\rho_{j}\right)^{2}\right) \\
\widetilde{H_{\bar{\rho}}}(\kappa) \equiv & \mathbf{F}^{-1}\left(\int_{\mathbb{R}^{d N}}^{\oplus} \widetilde{H}(p) d p\right) \mathbf{F} \\
= & \tilde{E}(D, \kappa) \otimes I+\kappa I \otimes H_{b},
\end{aligned}
$$


where $E^{R E N}(D, \kappa)$ and $\widetilde{E}(D, \kappa)$ are pseudo differential operators on $L^{2}\left(\mathbb{R}^{d N}\right)$ with symbols $E^{R E N}(p, \kappa)$ and $\tilde{E}(p, \kappa)$ respectively.

Theorem 3.2 Suppose $\vec{\rho} \in \widetilde{P}$. Then $H_{\vec{\rho}}^{R E N}(\kappa)$ and $\widetilde{H}_{\bar{\rho}}(\kappa)$ are essentially self-adjoint on any core of $H_{0}$ and bounded from below.

Proof: By the definition of $E^{R E N}(D, \kappa)$ and $\widetilde{E}(D, \kappa), H_{\vec{\rho}}{ }^{R E N}(\kappa)$ and $\widetilde{H_{\bar{\rho}}}(\kappa)$ are symmetric. For $f \in D(-\Delta)$, there exist $d_{1}(\kappa)$ and $d_{2}(\kappa)$ such that

$$
\begin{aligned}
& \|\tilde{E}(D, \kappa) f\|_{L^{2}\left(\mathbb{R}^{d N}\right)} \leq d_{1}(\kappa)\|-\Delta f\|_{L^{2}\left(\mathbb{R}^{d N}\right)} \\
& \left\|E^{R E N}(D, \kappa) f\right\|_{L^{2}\left(\mathbb{R}^{d N}\right)} \leq d_{2}(\kappa)\|-\Delta f\|_{L^{2}\left(\mathbb{R}^{d N}\right)} .
\end{aligned}
$$

Hence, similar to the proof of Theorem 2.2, the Nelson commutator theorem yields desired results.

\section{Remark 3.3 Write}

$$
E(p, \kappa)=\frac{\hbar^{2} \kappa^{2}}{2 m} p^{2}+\sum_{\mu=1}^{d} \sum_{i=1}^{N} \frac{\hbar^{2} \kappa^{2}}{m} p_{\mu}^{i} \tilde{p}_{\mu}^{i}(\kappa)+\sum_{\mu=1}^{d} \sum_{i=1}^{N} \frac{\hbar^{2} \kappa^{2}}{2 m} \tilde{p}_{\mu}^{i}(\kappa)^{2}+\square(\kappa) .
$$

Then the first and second terms on the right hand side of (3.6) diverge as $\kappa \rightarrow \infty$ for $p \neq 0$, but the rest terms not. Actually we see that

$$
\begin{aligned}
\lim _{\kappa \rightarrow \infty} \frac{\hbar^{2} \kappa^{2}}{2 m} \sum_{\mu=1}^{d} \sum_{i=1}^{N} \tilde{p}_{\mu}^{i}(\kappa)^{2} & =\frac{1}{2 m}\left(\frac{e^{2}}{2 m c^{2}}\right)\left(\frac{d-1}{d}\right)^{2} \sum_{\alpha=1}^{d} \sum_{k=1}^{N}\left(\sum_{j=1}^{N} \hbar p_{\alpha}^{j}\left\langle\frac{\hat{\rho}_{j}}{\sqrt{\omega^{3}}}, \frac{\hat{\rho}_{k}}{\sqrt{\omega}}\right\rangle_{L^{2}\left(\mathbb{R}^{d}\right)}\right)^{2} \\
& \equiv E^{\infty}(p) .
\end{aligned}
$$

Then, by (3.2), concerning an asymptotic behavior of $H_{\vec{\rho}}(\kappa)$ as $\kappa \rightarrow \infty$, we should subtract the first and second terms in the right hand side of (3.6) from the original Hamiltonian $H_{\vec{\rho}}(\kappa)$. However one can not say that $\bar{p}_{\mu}^{i}(\kappa)^{2}$ is real and nonnegative for any $p \in \mathbb{R}^{d N}$. To guarantee the nonnegative self-adjointness of the Hamiltonian $H_{\vec{\rho}}{ }^{R E N}(p, \kappa)$ with the divergence terms subtracted, we should define $\widetilde{E}(p, \kappa)$ such as (3.5). In this sense, we may say that the operator $H_{\vec{\rho}}^{R E N}(\kappa)$ has an interpretation of the Hamiltonian $H_{\vec{\rho}}(\kappa)$ with the infinite self-energy of the nonrelativistic particles subtracted. 
We define

$$
\mathcal{U}(\kappa)=\mathbf{F}^{-1}\left(\int_{\mathbb{R}^{d N}}^{\oplus} \mathcal{U}(\kappa, p) d p\right) \mathbf{F}
$$

Then we have the following theorem.

Theorem 3.4 Suppose that $\vec{\rho} \in \tilde{P}$. Then

$$
\begin{aligned}
s-\lim _{\kappa \rightarrow \infty} \mathcal{U}(\kappa) & =\exp \left(\sum_{j=1}^{N} \frac{e \hbar}{m} D_{\mu}^{j} \otimes\left\{a\left(\oplus_{r=1}^{d-1} \frac{e_{\mu}^{r} \hat{\rho}_{j}}{\sqrt{2 \hbar c^{3} \omega^{3}}}\right)-a^{\dagger}\left(\oplus_{r=1}^{d-1} \frac{e_{\mu}^{r} \hat{\rho}_{j}}{\sqrt{2 \hbar c^{3} \omega^{3}}}\right)\right\}\right) \\
& \equiv \mathcal{U}(\infty)
\end{aligned}
$$

Proof: From [6,Theorem 3.11] it follows (3.7).

We take scalar potentials $V$ to be real-valued measurable functions on $\mathbb{R}^{d N}$ and put

$$
C_{\kappa}(V)=\mathcal{U}^{-1}(\kappa)(V \otimes I) \mathcal{U}(\kappa), \quad C(V)=\mathcal{U}^{-1}(\infty)(V \otimes I) \mathcal{U}(\infty)
$$

We introduce conditions $(\mathbf{V}-\mathbf{1})$ and $(\mathbf{V}-\mathbf{2})$ as follows.

(V-1) For sufficiently large $\kappa>0, D(\widetilde{E}(D, \kappa)) \subset D(V)$ and for $\lambda>0, V(\widetilde{E}(D, \kappa)+\lambda)^{-1}$ is bounded with

$$
\lim _{\lambda \rightarrow \infty}\left\|V(\tilde{E}(D, \kappa)+\lambda)^{-1}\right\|=0
$$

where the convergence is uniform in sufficiently large $\kappa>0$.

(V-2) For $\lambda>0, V(\tilde{E}(D, \kappa)+\lambda)^{-1}$ is strongly continuous in $\kappa$ and

$$
s-\lim _{\kappa \rightarrow \infty} V(\tilde{E}(D, \kappa)+\lambda)^{-1}=V\left(E^{\infty}(D)+\lambda\right)^{-1}
$$

The condition (3.9) yields that, by the Kato-Rellich theorem and commutativity of $\mathcal{U}(\kappa)$ and $(\tilde{E}(D, \kappa)+\lambda)^{-1}$, operators $\tilde{E}(D, \kappa) \otimes I+C_{\kappa}(V)$ are essentially self-adjoint on any core of $D(\widetilde{E}(D, \kappa) \otimes I)$ and uniformly bounded from below in sufficiently large $\kappa>0$. Moreover since $I \otimes H_{b}$ is nonnegative and commute with $\widetilde{E}(D, \kappa) \otimes I$, one can see that

$$
\widetilde{H}_{\vec{\rho}}(V, \kappa) \equiv \tilde{E}(D, \kappa) \otimes I+C_{\kappa}(V)+\kappa I \otimes H_{b}
$$


is essentially self-adjoint on any core of $D\left(\tilde{E}(D, \kappa) \otimes I+\kappa I \otimes H_{b}\right)$ and uniformly bounded from below in sufficiently large $\kappa>0$. In particular, $D\left(H_{0}\right)$ is a core of $\widetilde{H_{\vec{\rho}}}(V, \kappa)$. Put

$$
H_{\vec{\rho}}^{R E N}(V, \kappa) \equiv H_{\vec{\rho}}^{R E N}(\kappa)+V \otimes I
$$

Theorem 3.5 Let $\vec{\rho} \in \widetilde{P}$. Suppose that $V$ satisfies $(\mathbf{V}-\mathbf{1})$ and $(\mathbf{V}-\mathbf{2})$. Then, for sufficiently large $\kappa>0$, the operator $H_{\bar{\rho}}^{R E N}(V, \kappa)$ is essentially self-adjoint on $D\left(H_{0}\right)$ and bounded from below uniformly in sufficiently large $\kappa>0$. Moreover the unitary operator $\mathcal{U}(\kappa)$ maps $D\left(H_{0}\right)$ onto itself and for $z \in \mathbb{C} \backslash \mathbb{R}$ or $z<0$ with $|z|$ sufficiently large,

$$
\left(H_{\vec{\rho}}^{R E N}(V, \kappa)-z\right)^{-1}=\mathcal{U}(\kappa)\left(\widetilde{H_{\vec{\rho}}}(V, \kappa)-z\right)^{-1} \mathcal{U}^{-1}(\kappa)
$$

Proof: Since $\mathcal{U}(\kappa)$ maps $D\left(I \otimes H_{b}\right)$ onto itself (see Theorem 3.1) and $-\Delta \otimes I$ commutes with $\mathcal{U}(\kappa)$ on $D(-\Delta \otimes I), \mathcal{U}(\kappa)$ maps $D\left(H_{0}\right)$ onto itself. Put

$$
S_{0}^{\infty}\left(\mathbb{R}^{d N}\right)=\left\{f \in L^{2}\left(\mathbb{R}^{d N}\right) \mid \hat{f} \in C_{0}^{\infty}\left(\mathbb{R}^{d N}\right)\right\}
$$

At first, by Theorem 3.1, we see that for $\Phi \in S_{0}^{\infty}\left(\mathbb{R}^{d N}\right) \widehat{\otimes} D\left(H_{b}\right)$,

$$
H_{\vec{\rho}}^{R E N}(V, \kappa) \Phi=\mathcal{U}(\kappa) \widetilde{H_{\vec{\rho}}}(V, \kappa) \mathcal{U}^{-1}(\kappa) \Phi
$$

By a limiting argument we can extend (3.11) to $\Phi \in D\left(H_{0}\right)$. Since $D\left(H_{0}\right)$ is a core of $\widetilde{H_{\vec{\rho}}}(V, \kappa)$ and $\mathcal{U}(\kappa)$ maps $D\left(H_{0}\right)$ onto itself, the right hand side of $(3.11)$ is essentially self-adjoint on $D\left(H_{0}\right)$. So is the left hand side of (3.11). (3.10) can be easily shown.

We want to consider a scaling limit of $H_{\vec{\rho}}^{R E N}(V, \kappa)$ as $\kappa \rightarrow \infty$. In [5], a general theory of the strong resolvent limit of self-adjoint operators including abstract versions like as the selfadjoint operator $\widetilde{H_{\vec{\rho}}}(V, \kappa)$ has been established. We shall apply the theory in [5] with a little modification. Let $V$ satisfy $(\mathbf{V}-\mathbf{1})$. Then since $D(C(V)) \supset D(-\Delta) \widehat{\otimes} D\left(H_{b}\right)$, one can define, for $\Phi \in \mathcal{F}(\mathcal{W})$ and $\Psi \in D\left(H_{b}\right)$, a symmetric operator $E_{\Phi, \Psi}(C(V))$ with $D\left(E_{\Phi, \Psi}(C(V))=\right.$ $D(-\Delta)$ by

$$
\left\langle f, E_{\Phi, \Psi}(C(V)) g\right\rangle_{L^{2}\left(\mathbb{R}^{d N}\right)}=\langle f \otimes \Phi, C(V)(g \otimes \Psi)\rangle_{\mathcal{F}}, \quad f \in L^{2}\left(\mathbb{R}^{d N}\right), g \in D(-\Delta)
$$


In particular, we call $E_{\Omega, \Omega}(C(V)) \equiv E_{\Omega}(C(V))$ "the partial expectation of $C(V)$ with respect to $\Omega$ " $([5$, Section II $])$.

Theorem 3.6 Let $\vec{\rho} \in \tilde{P}$. Suppose that $V$ satisfies the conditions $(\mathbf{V}-\mathbf{1})$ and $(\mathbf{V}-\mathbf{2})$. Then for $z \in \mathbb{C} \backslash \mathbb{R}$ or $z<0$ with $|z|$ sufficiently large,

$$
s-\lim _{\kappa \rightarrow \infty}\left(H_{\bar{\rho}}^{R E N}(V, \kappa)-z\right)^{-1}=\mathcal{U}(\infty)\left\{\left(E^{\infty}(D)+E_{\Omega}(C(V))-z\right)^{-1} \otimes P_{0}\right\} \mathcal{U}^{-1}(\infty)
$$

where $P_{0}$ is the projection from $\mathcal{F}(\mathcal{W})$ to the one dimensional subspace $\{\alpha \Omega \mid \alpha \in \mathbb{C}\}$.

Proof: By $(\mathbf{V}-\mathbf{1})$ and $(\mathbf{V}-\mathbf{2})$, we see that

$(\mathrm{V}-\mathbf{1})$ ' For sufficiently large $\kappa>0, D(\tilde{E}(D, \kappa)) \subset D\left(C_{\kappa}(V)\right)$ and for $\lambda>0$, $C_{\kappa}(V)(\widetilde{E}(D, \kappa)+\lambda)^{-1}$ is bounded with

$$
\lim _{\lambda \rightarrow \infty}\left\|C_{k}(V)(\tilde{E}(D, \kappa)+\lambda)^{-1}\right\|=0
$$

where the convergence is uniform in sufficiently large $\kappa>0$.

$(\mathbf{V - 2})^{\prime}$ For $\lambda>0, C_{\kappa}(V)(\tilde{E}(D, \kappa)+\lambda)^{-1}$ is strongly continuous in $\kappa$ and

$$
s-\lim _{\kappa \rightarrow \infty} C_{k}(V)(\tilde{E}(D, \kappa)+\lambda)^{-1}=C(V)\left(E^{\infty}(D)+\lambda\right)^{-1}
$$

From $\left[5\right.$, Section II], $(\mathbf{V}-\mathbf{1})^{\prime}$ and $(\mathbf{V}-\mathbf{2})^{\prime}$ imply that

$$
s-\lim _{\kappa \rightarrow \infty}\left(\widetilde{H_{\vec{\rho}}}(V, \kappa)-z\right)^{-1}=\left(E^{\infty}(D)+E_{\Omega}(C(V))-z\right)^{-1} \otimes P_{0}
$$

Thus by Theorems 3.4 and 3.5 , we get (3.12).

We want to see $E_{\Omega}(C(V))$ more explicitly. For $\vec{\rho} \in \tilde{P}$, let $\tilde{\Delta}^{\infty}=\left(\tilde{\Delta}_{i j}^{\infty}\right)_{1 \leq i, j \leq d}$, where $\tilde{\Delta}_{i j}^{\infty}$ is defined in (1.3). Let $\mathbf{I}_{d \times d}$ denote $d \times d$-identity matrix. Since $\Delta^{\infty} \equiv \tilde{\Delta}^{\infty} \otimes \mathbf{I}_{d \times d} \epsilon$ 
$M(N) \otimes N(d) \cong M(d N)$ is a nonnegative symmetric matrix, there exist unitary matrices $\mathbf{T} \in M(d N)$ so that

$$
\mathbf{T} \Delta^{\infty} \mathbf{T}^{-1}=\left(\begin{array}{cccc}
\lambda_{1} \mathbf{I}_{d \times d} & & & \\
& \lambda_{2} \mathbf{I}_{d \times d} & & \\
& & \ddots & \\
& & & \lambda_{N} \mathbf{I}_{d \times d}
\end{array}\right)
$$

where $\lambda_{1} \geq \lambda_{2} \ldots \geq \lambda_{N} \geq 0$.

Theorem 3.7 Suppose $\lambda_{1} \geq \lambda_{2} \ldots \geq \lambda_{K}>0, \lambda_{K+1}=\ldots=\lambda_{N}=0$ and fix a unitary operator $\mathrm{T}$ in (3.13). Let $x=\left(x_{1}, \ldots, x_{N}\right), x_{j} \in \mathbb{R}^{d}, j=1, \ldots, N$ and $V$ satisfy

$$
\int_{\mathbb{R}^{d K}} d y_{1} \ldots d y_{K}|V| \circ \mathbf{T}^{-1}\left(y_{1}, \ldots, y_{K},(\mathbf{T} x)_{K+1}, \ldots,(\mathbf{T} x)_{N}\right) \exp \left(-\frac{\sum_{j=1}^{K}\left|(\mathbf{T} x)_{j}-y_{j}\right|^{2}}{2 \lambda_{1} \ldots \lambda_{K}}\right)<\infty
$$

Moreover we suppose that the left hand side of (3.14) is locally bounded. Then the partial expectation $E_{\Omega}(C(V))$ is given by a multiplication operator $V_{\text {eff }}$;

$$
\begin{gathered}
V_{e f f}(x)=\left(2 \pi \lambda_{1} \ldots \lambda_{K}\right)^{-\frac{d}{2}} \int_{\mathbb{R}^{d K}} d y_{1} \ldots d y_{K} V \circ \mathbf{T}^{-1}\left(y_{1}, \ldots, y_{K},(\mathbf{T} x)_{K+1}, \ldots,(\mathbf{T} x)_{N}\right) \\
\times \exp \left(-\frac{\sum_{j=1}^{K}\left|(\mathbf{T} x)_{j}-y_{j}\right|^{2}}{2 \lambda_{1} \ldots \lambda_{K}}\right)
\end{gathered}
$$

In particular, in the case where $\tilde{\Delta}^{\infty}$ is non-degenerate, $V_{\text {eff }}$ is given by

$$
V_{e f f}(x)=\left(2 \pi \operatorname{det} \tilde{\Delta}^{\infty}\right)^{-\frac{d}{2}} \int_{\mathbb{R}^{d N}} V(y) \exp \left(-\frac{|x-y|^{2}}{2 \operatorname{det} \tilde{\Delta}^{\infty}}\right) d y .
$$

Proof: Suppose $V \in \mathcal{S}\left(\mathbb{R}^{d N}\right)$, which is the set of the rapidly decreasing infinitely continuously differentiable functions on $\mathbb{R}^{d N}$. Then the direct calculation shows that for $f, g \in L^{2}\left(\mathbb{R}^{d N}\right)$

$$
\left\langle f, E_{\Omega}(C(V)) g\right\rangle_{L^{2}\left(\mathbb{R}^{d N}\right)}=\left\langle f, V_{e f f} g\right\rangle_{L^{2}\left(\mathbb{R}^{d N}\right)}
$$

We next consider the case where $V$ is bounded. In this case we can approximate $V$ by a sequence $\left\{V_{n}\right\}_{n=1}^{\infty}, V_{n} \in \mathcal{S}\left(\mathbb{R}^{d N}\right)$, such that

$$
\left\|V-V_{n}\right\|_{\infty} \rightarrow 0(n \rightarrow \infty)
$$


where $\|\cdot\|_{\infty}$ denotes the sup norm. Then we have

$$
E_{\Omega}\left(C\left(V_{n}\right)\right) \rightarrow E_{\Omega}(C(V))(n \rightarrow \infty)
$$

strongly. Moreover $\left(V_{n}\right)_{e f f}(x) \rightarrow V_{\text {eff }}(x)$ for all $x \in \mathbb{R}^{d N}$. Thus for $f, g \in L^{2}\left(\mathbb{R}^{d N}\right)$,

follows for such $V$. Finally, let $V$ satisfy (3.14). Define

$$
V_{n}= \begin{cases}V(x) & |V(x)| \leq n, \\ n & |V(x)|>n .\end{cases}
$$

Hence for $f \in L^{2}\left(\mathbb{R}^{d N}\right)$ and $g \in D(-\Delta)$, we have

$$
\left\langle f, E_{\Omega}\left(C\left(V_{n}\right)\right) g\right\rangle_{L^{2}\left(\mathbb{R}^{d N}\right)} \rightarrow\left\langle f, E_{\Omega}(C(V)) g\right\rangle_{L^{2}\left(\mathbb{R}^{d N}\right)}(n \rightarrow \infty)
$$

On the other hand, since the left hand side of (3.14) is locally bounded, we can see that for $f \in C_{0}^{\infty}\left(\mathbb{R}^{d N}\right)$ and $g \in D(-\Delta)$,

$$
\left\langle f,\left(V_{n}\right)_{e f f} g\right\rangle_{L^{2}\left(\mathbb{R}^{d N}\right)} \rightarrow\left\langle f, V_{e f f} g\right\rangle_{L^{2}\left(\mathbb{R}^{d N}\right)}(n \rightarrow \infty)
$$

which completes the proof.

Remark 3.8 In Theorem 3.7, in the case where $\widetilde{\Delta}^{\infty}$ is non-degenerate, since the left hand side of (3.14) is continuous in $x \in \mathbb{R}^{d N}$, it is necessarily locally bounded.

We call $V_{\text {eff }}$ "the effective potential with respect to $V$ ". We give some examples of scalar potentials $V$ and ultraviolet cut off functions $\rho$.

Example 3.9 ([non-degenerate case $]$ ) Let

$$
\tilde{\Delta}_{i j}^{\infty}=\delta_{i j} \frac{1}{2} \frac{d-1}{d}\left(\frac{\hbar}{m c}\right)^{2} \frac{e^{2}}{\hbar c} \int_{\mathbb{R}^{d}} d k \frac{\hat{\rho}_{i}(k)^{2}}{\omega(k)^{3}} .
$$

Then there exist positive constants $\delta_{1}$ and $\delta_{2}$ such that for sufficiently large $\kappa>0$

$$
\delta_{1}|p|^{2} \leq \widetilde{E}(p, \kappa) \leq \delta_{2}|p|^{2}
$$


Let $d=3$ and $V$ be the Coulomb potential;

$$
V\left(x_{1}, \ldots, x_{N}\right)=-\sum_{j=1}^{N} \frac{\alpha_{j}}{\left|x_{j}\right|}+\sum_{i \neq j} \frac{\beta_{i j}}{\left|x_{i}-x_{j}\right|}, \quad \alpha_{j} \geq 0, \beta_{i j} \geq 0 .
$$

Then $V$ is the Kato class potential ([10], Theorem X.16). Namely for any $\epsilon>0$, there exists $b \geq 0$ such that $D(V) \supset D(-\Delta)$ and

$$
\|V \Phi\|_{L^{2}\left(\mathbb{R}^{3 N}\right)} \leq \epsilon\|-\Delta \Phi\|_{L^{2}\left(\mathbb{R}^{3 N}\right)}+b\|\Phi\|_{L^{2}\left(\mathbb{R}^{3 N}\right)}
$$

Together with (3.16) and (3.17), one can see that $V$ satisfies $(\mathbf{V}-\mathbf{1}),(\mathbf{V}-\mathbf{2})$ and for any $t>0$

$$
\int_{\mathbb{R}^{3 d}}|V|(y) e^{-t|x-y|^{2}} d y<\infty
$$

Then the scaling limit of the Pauli-Fierz Hamiltonian with the Coulomb potential exists and has the effective potential given by

$$
\begin{aligned}
V_{e f f}(x) & =(2 \pi \gamma)^{-\frac{3}{2}} \int_{\mathbb{R}^{3 N}} V(y) e^{-\frac{|x-y|^{2}}{2 \gamma}} d y \\
\gamma & =\left\{\frac{1}{3}\left(\frac{\hbar}{m c}\right)^{2} \frac{e^{2}}{\hbar c}\right\}^{N} \Pi_{j=1}^{N}\left(\int_{\mathbb{R}^{3}} d k \frac{\hat{\rho}_{j}^{2}(k)}{\omega(k)^{3}}\right) .
\end{aligned}
$$

Moreover

$$
E^{\infty}(D)=-\frac{1}{2 m}\left(\frac{e^{2}}{2 m c^{2}}\right)\left(\frac{d-1}{d}\right)^{2} \otimes_{j=1}^{N}\left\|\frac{\hat{\rho}_{j}}{\omega}\right\|^{4} \hbar^{2} \Delta_{j},
$$

where $\Delta_{j}, j=1, \ldots, N$, is the Laplacian in $L^{2}\left(\mathbb{R}^{d}\right)$.

Example 3.10 ([non-degenerate case]) Let $\widetilde{\Delta}^{\infty}$ be non-degenerate and $V$ be the Phillips perturbation with respect to $-\Delta([12])$. Then (3.16) holds with some $\delta_{1}$ and $\delta_{2}$. Hence $V$ satisfies $(\mathbf{V}-\mathbf{1}),(\mathbf{V}-\mathbf{2})$ and for any $t>0$

$$
\int_{\mathbb{R}^{d N}}|V|(y) e^{-t|x-y|^{2}} d y<\infty
$$

Hence the scaling limit of the Pauli-Fierz Hamiltonian with Phillips perturbation exists and has the effective potential in Theorem 3.7. 
Example 3.11 ([degenerate case]) Let $V$ be a real-valued bounded function. Then $V$ satisfies the conditions $(V-1)$ and $(V-2)$. Hence the scaling limit of the Pauli-Fierz Hamiltonian with the scalar potential $V$ exists for all $\vec{\rho} \in \tilde{P}$.

Example 3.12 ([degenerate case $]$ ) Let $\rho_{i}=\rho, i=1, \ldots, N$ and $V \operatorname{satisfy}(\mathbf{V}-\mathbf{1}),(\mathbf{V}-\mathbf{2})$ and the assumption stated in Theorem 3.7. Then rank $\widetilde{\Delta}^{\infty}=1$ and the non-zero eigenvalue $C$ is given by

$$
C=\frac{N}{2} \frac{d-1}{d}\left(\frac{\hbar}{m c}\right)^{2} \frac{e^{2}}{\hbar c} \int_{\mathbb{R}^{d}} d k \frac{\hat{\rho}(k)^{2}}{\omega(k)^{3}}
$$

Thus the scaling limit of the Pauli-Fierz Hamiltonian with the ultraviolet cut-off function $\rho$ exists and has the following effective potential:

$$
V_{e f f}(x)=(2 \pi C)^{-\frac{d}{2}} \int_{\mathbb{R}^{d}} d y_{1} V \circ \mathbf{T}^{-1}\left(y_{1},(\mathbf{T} x)_{2}, \ldots,(\mathbf{T} x)_{N}\right) \exp \left(-\frac{\left|(\mathbf{T} x)_{1}-y_{1}\right|^{2}}{2 C}\right) .
$$

Moreover

$$
E^{\infty}(D)=-\frac{1}{2 m}\left(\frac{N^{2} e^{2}}{2 m c^{2}}\right)\left(\frac{d-1}{d}\right)^{2}\left\|\frac{\hat{\rho}}{\omega}\right\|^{4} \hbar^{2} \Delta
$$

where $\Delta$ is the Laplacian in $L^{2}\left(\mathbb{R}^{d N}\right)$.

\section{CONCLUDING REMARK}

As is seen in Theorem 3.7, the effective potential $V_{\text {eff }}$ is characterized by the matrix-valued functional $\widetilde{\Delta}^{\infty}=\widetilde{\Delta}^{\infty}(\vec{\rho})$, which has the following mathematical meaning; putting

$$
\mathcal{U}(\infty)\left(x_{i} \otimes I\right) \mathcal{U}^{-1}(\infty)-x_{i} \otimes I \equiv \Delta x_{i}, \quad i=1, \ldots, N
$$

we see that the partial expectation of $\Delta x_{i} \Delta x_{j}$ with respect to $\Omega$ is as follows;

$$
E_{\Omega}\left[\left(\Delta x_{i} \Delta x_{j}\right)\right]=\tilde{\Delta}_{i j}^{\infty}(\vec{\rho}) I
$$


In one-nonrelativistic particle case, A.Arai [5] shows that the partial expectation $E_{\Omega}\left[(\Delta x)^{2}\right]$ with respect to $\Omega$ may be interpreted as the mean square fluctuation in position of onenonrelativistic particle ([2]). In this sense, $\widetilde{\Delta}_{i j}^{\infty}(\vec{\rho})$ may also be interpreted as correlation of fluctuations in position of the $i$-th and the $j$-th nonrelativistic particles under the action of quantized radiation fields. 


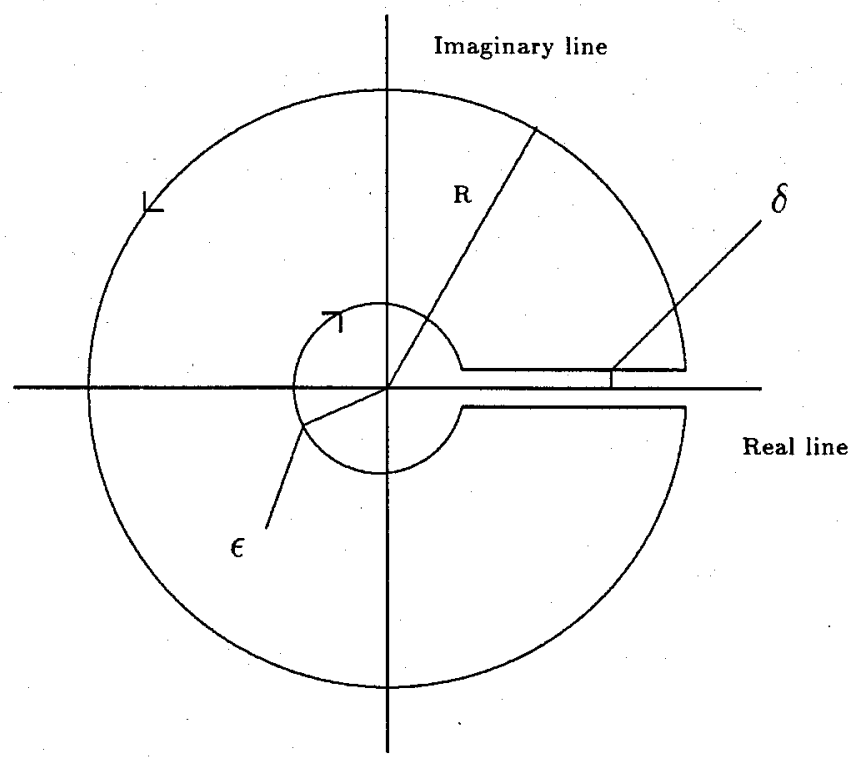

Figure 1: Cut Plane $\mathbb{C}_{R, \delta, \epsilon}$

\section{APPENDIX}

In this Appendix we prove Lemma 2.6. For simplicity, in this proof, we omit $\kappa$ in notations and put

$$
\lambda=\frac{e^{2}}{c^{2}}, \quad \widehat{G}=\omega^{1-\frac{d}{2}} G \omega^{1-\frac{d}{2}}, \quad \widehat{G}_{t}=\omega^{1-\frac{d}{2}} G_{t} \omega^{1-\frac{d}{2}}, t>0
$$

(1) : This follows from the definition of $T_{\mu \nu}$ and $T_{\mu \nu}^{*}$, and Definition 2.1 (4).

(2) :For $f \in M_{-1}$,

$$
\begin{aligned}
\left\langle\omega^{-\frac{3}{2}} Q_{i}, \omega^{-\frac{1}{2}} d_{\nu \alpha} T_{\mu \nu} f\right\rangle_{L^{2}\left(\mathbb{R}^{d}\right)}= & \left\langle d_{\mu \alpha} \omega^{-\frac{3}{2}} Q_{i}, \omega^{-\frac{1}{2}} f\right\rangle_{L^{2}\left(\mathbb{R}^{d}\right)} \\
& +\sum_{j=1}^{N}\left\langle d_{\nu \alpha} \omega^{-\frac{3}{2}} Q_{i}, \lambda \omega^{-\frac{1}{2}} Q_{j} \widehat{G} d_{\mu \nu} \hat{\rho}_{j} f\right\rangle_{L^{2}\left(\mathbb{R}^{d}\right)} \\
= & I+I I
\end{aligned}
$$

Using (2.8), one can see that

$$
I I=\lim _{t \rightarrow 0} \lambda \sum_{j=1}^{N} \int \frac{\bar{Q}_{i}(k) Q_{j}(k) d_{\mu \nu}\left(k^{\prime}\right) d_{\nu \alpha}(k) \hat{\rho}_{j}\left(k^{\prime}\right) f\left(k^{\prime}\right)}{\left(k^{2}-k^{\prime 2}+i t\right) k^{2}} d k d k^{\prime}
$$




$$
\begin{aligned}
& =\lim _{t \rightarrow 0} \lambda \sum_{j=1}^{N} \frac{d-1}{d} \mathbf{V}_{d} \int \frac{\sum_{k, l=1}^{N} D_{-i k}^{-1}(s) D_{+j l}^{-1}(s) \hat{\rho}_{k}(\sqrt{s}) \hat{\rho}_{l}(\sqrt{s}) s^{\frac{d}{2}-1} F_{j}\left(k^{\prime}\right)}{\left(s-k^{\prime 2}+i t\right) s} d s d k^{\prime} \\
& =\lim _{t \rightarrow 0} \frac{1}{2 \pi i} \sum_{j=1}^{N} \int \frac{1}{\left(s-k^{2}+i t\right) s}\left(D_{-i j}^{-1}(s)-D_{+j i}^{-1}(s)\right) F_{j}\left(k^{\prime}\right) d s d k^{\prime}
\end{aligned}
$$

where $F_{j}\left(k^{\prime}\right)=d_{\mu \alpha}\left(k^{\prime}\right) \hat{\rho}_{j}\left(k^{\prime}\right) f\left(k^{\prime}\right)$. Using the contour integral on the cut plane $\mathbb{C}_{R, \delta, \epsilon}$ (Figure 1), by (2.5) and (2.6), we have

$$
\begin{aligned}
& \frac{1}{2 \pi i} \int_{0}^{\infty} \frac{1}{\left(s-k^{2}+i t\right) s}\left(D_{-i j}^{-1}(s)-D_{+j i}^{-1}(s)\right) d s \\
& =\lim _{\epsilon \rightarrow 0} \frac{1}{2 \pi i} \int_{\epsilon}^{\infty} \frac{1}{\left(s-k^{\prime 2}+i t\right) s}\left(D_{-i j}^{-1}(s)-D_{+j i}^{-1}(s)\right) d s \\
& =\lim _{\epsilon \rightarrow 0} \lim _{R \rightarrow \infty, \delta \rightarrow 0}-\frac{1}{2 \pi i} \int_{\mathbb{C}_{R, \delta, \epsilon}} \frac{D_{i j}^{-1}(z)}{\left(z-k^{\prime 2}+i t\right) z} d z-\frac{D_{i j}^{-1}(0)}{-k^{\prime 2}+i t} \\
& =-\frac{D_{i j}^{-1}\left(k^{\prime 2}-i t\right)}{k^{\prime 2}-i t}-\frac{D_{i j}^{-1}(0)}{-k^{\prime 2}+i t} .
\end{aligned}
$$

Then

$$
\begin{aligned}
I I & =\lim _{t \rightarrow 0} \sum_{j=1}^{N} \int \frac{F_{j}\left(k^{\prime}\right) D_{i j}^{-1}(0)}{k^{2}-i t}-\frac{D_{i j}^{-1}\left(k^{\prime 2}-i t\right) F_{j}\left(k^{\prime}\right)}{k^{\prime 2}-i t} d k^{\prime} \\
& =-\left\langle d_{\mu \alpha} \omega^{-\frac{3}{2}} Q_{i}, \omega^{-\frac{1}{2}} f\right\rangle_{L^{2}\left(\mathbb{R}^{d}\right)}+\left\langle d_{\mu \alpha} \omega^{-\frac{3}{2}} \sum_{j=1}^{N} D_{i j}^{-1}(0) \hat{\rho}_{j}, \omega^{-\frac{1}{2}} f\right\rangle_{L^{2}\left(\mathbb{R}^{d}\right)}
\end{aligned}
$$

Hence we get (2).

(3),(4) : They are direct calculations.

(5) : For $f, g \in M_{0}$,

$$
\begin{aligned}
<T_{\mu \nu}^{*} d_{\nu \alpha} T_{\alpha \beta} f, g>_{L^{2}\left(\mathbb{R}^{d}\right)}= & <d_{\mu \beta} f, g>_{L^{2}\left(\mathbb{R}^{d}\right)}+\lambda \sum_{j=1}^{N}<d_{\nu \beta} f, Q_{i} \widehat{G} d_{\mu \nu} \hat{\rho}_{i} g>_{L^{2}\left(\mathbb{R}^{d}\right)} \\
& +\lambda \sum_{j=1}^{N}<d_{\mu \alpha} Q_{j} \widehat{G} d_{\alpha \beta} \hat{\rho}_{j} f, g>_{L^{2}\left(\mathbb{R}^{d}\right)} \\
& +\lambda^{2} \frac{d-1}{d} \sum_{i, j=1}^{N}<Q_{i} \widehat{G} d_{\alpha \beta} \hat{\rho}_{i} f, Q_{j} \widehat{G} d_{\mu \alpha} \hat{\rho}_{j} g>_{L^{2}\left(\mathbb{R}^{d}\right)} \\
= & I+I I+I I I+I V .
\end{aligned}
$$


Then,

$$
\begin{aligned}
I V & =\lim _{t \rightarrow 0} \lambda^{2} \sum_{i, j, k, l=1}^{N} \mathbf{V}_{d} \frac{d-1}{d} \int \frac{D_{-i k}^{-1}(s) D_{+j l}^{-1}(s) \hat{\rho}_{k}(\sqrt{s}) \hat{\rho}_{l}(\sqrt{s}) s^{\frac{d}{2}-1} F_{i j}\left(k^{\prime}, k^{\prime \prime}\right)}{\left(s-k^{\prime 2}-i t\right)\left(s-k^{\prime 2}+i t\right)} d s d k^{\prime} d k^{\prime \prime}, \\
& \equiv \lim _{t \rightarrow 0} I V_{t},
\end{aligned}
$$

where $F_{i j}\left(k^{\prime}, k^{\prime \prime}\right)=d_{\mu \alpha}\left(k^{\prime \prime}\right) \hat{\rho}_{j}\left(k^{\prime \prime}\right) d_{\alpha \beta}\left(k^{\prime}\right) \hat{\rho}_{i}\left(k^{\prime}\right) \bar{f}\left(k^{\prime}\right) g\left(k^{\prime \prime}\right)$. By using the cut plane integral method as in (2), we have

$$
\begin{aligned}
I V_{t}= & \sum_{i, j=1}^{N} \int \frac{-\lambda D_{i j}^{-1}\left(k^{\prime}+i t\right) F_{i j}\left(k^{\prime}, k^{\prime \prime}\right)}{k^{\prime 2}-k^{\prime 2}+2 i t}+\frac{-\lambda D_{i j}^{-1}\left(k^{\prime \prime}-i t\right) F_{i j}\left(k^{\prime}, k^{\prime \prime}\right)}{k^{\prime \prime 2}-k^{\prime 2}-2 i t} d k^{\prime} d k^{\prime \prime} \\
= & -\lambda \sum_{i, j=1}^{N}\left\langle d_{\alpha \beta} f, D_{j i}^{-1}(\cdot+i t) \hat{\rho}_{i} \widehat{G}_{2 t} d_{\mu \alpha} \hat{\rho}_{j} g\right\rangle_{L^{2}\left(\mathbb{R}^{d}\right)} \\
& -\lambda \sum_{i, j=1}^{N}\left\langle d_{\mu \alpha} D_{i j}^{-1}(\cdot+i t) \hat{\rho}_{j} \widehat{G}_{2 t} d_{\alpha \beta} \hat{\rho}_{i} f, g\right\rangle_{L^{2}\left(\mathbb{R}^{d}\right)} .
\end{aligned}
$$

By a limiting argument as $t \rightarrow 0$, we get

$$
\lim _{t \rightarrow 0} I V_{t}=-I I-I I I
$$

(6) : For $f, g \in M_{0}$,

$$
\begin{aligned}
\left\langle e_{\mu}^{r} T_{\mu \nu} d_{\nu \alpha} T_{\alpha \beta} e_{\beta}^{s} f, g\right\rangle_{L^{2}\left(\mathbb{R}^{d}\right)}= & <\delta_{r s} f, g>_{L^{2}\left(\mathbb{R}^{d}\right)}-\lambda<e_{\beta}^{r} \rho_{j} \widehat{G} \bar{Q}_{j} e_{\beta}^{s} f, g>_{L^{2}\left(\mathbb{R}^{d}\right)} \\
& -\lambda<f, e_{\mu}^{s} \rho_{j} G \bar{Q}_{j} e_{\mu}^{r} g>_{L^{2}\left(\mathbb{R}^{d}\right)} \\
& +\lambda^{2}<d_{\mu \beta} \rho_{j} \widehat{G} \bar{Q}_{j} e_{\beta}^{s} f, \rho_{j} \widehat{G} \bar{Q}_{j} e_{\mu}^{r} g>_{L^{2}\left(\mathbb{R}^{d}\right)} \\
= & I-I I-I I I+I V .
\end{aligned}
$$

We see that

$$
\begin{aligned}
I V= & \lambda^{2} \lim _{\epsilon \rightarrow 0} \sum_{i, j=1}^{N} \mathbf{V}_{d} \frac{d-1}{d} \int \frac{\hat{\rho}_{j}(\sqrt{s}) \hat{\rho}_{i}(\sqrt{s}) s^{\frac{d}{2}-1} H_{i j}\left(k^{\prime}, k^{\prime \prime}\right)}{\left(s-k^{\prime 2}-i \epsilon\right)\left(s-k^{\prime \prime 2}+i \epsilon\right)} d s d k^{\prime} d k^{\prime \prime} \\
= & \lambda \lim _{\epsilon \rightarrow 0} \sum_{i, j=1}^{N} \int\left(\frac{D_{i j}\left(k^{\prime 2}+i \epsilon\right)}{k^{\prime 2}-k^{\prime \prime 2}+2 i \epsilon}+\frac{D_{i j}\left(k^{\prime \prime 2}-i \epsilon\right)}{k^{\prime 2}-k^{\prime 2}-2 i \epsilon}\right) H_{i j}\left(k^{\prime}, k^{\prime \prime}\right) d k^{\prime} d k^{\prime \prime} \\
= & \lambda \lim _{\epsilon \rightarrow 0} \sum_{i, j=1}^{N}<f, e_{\mu}^{s} Q_{j} D_{i j}(\cdot+i \epsilon) \widehat{G}_{2 \epsilon} \bar{Q}_{i} e_{\mu}^{r} g>_{L^{2}\left(\mathbb{R}^{d}\right)} \\
& +\lambda \sum_{i, j=1}^{N}<e_{\mu}^{r} Q_{i} \bar{D}_{i j}(\cdot-i \epsilon) \widehat{G}_{2 \epsilon} \bar{Q}_{j} e_{\mu}^{s} f, g>_{L^{2}\left(\mathbb{R}^{d}\right)},
\end{aligned}
$$


where $H_{i j}\left(k^{\prime}, k^{\prime \prime}\right)=Q_{j}\left(k^{\prime}\right) \bar{Q}_{i}\left(k^{\prime \prime}\right) e_{\mu}^{s}\left(k^{\prime}\right) e_{\mu}^{r}\left(k^{\prime \prime}\right) \bar{f}\left(k^{\prime}\right) g\left(k^{\prime \prime}\right)$. Note that $\sum_{j=1}^{N} Q_{j} D_{+i j}=\hat{\rho}_{i}$. Then

$$
\begin{aligned}
& =\lambda \sum_{i=1}^{N}<f, e_{\mu}^{s} \hat{\rho}_{i} \widehat{G} \bar{Q}_{i} e_{\mu}^{r} g>_{L^{2}\left(\mathbb{R}^{d}\right)}+\lambda \sum_{j=1}^{N}<e_{\mu}^{r} \hat{\rho}_{j} \widehat{G} \bar{Q}_{j} e_{\mu}^{s} f, g>_{L^{2}\left(\mathbb{R}^{d}\right)} \\
& =I I+I I I .
\end{aligned}
$$

Hence we get the desired results.

\section{REFERENCES}

[1]H.A.Bethe, The electromagnetic shift of energy levels, Phys.Rev.72,(1947)339-342.

[2] T.A.Welton, Some observable effects of the quantum mechanical fluctuations of the electromagnetic field, Phys.Rev.74(1948)1157-1167.

[3] A.Arai, A note on scattering theory in non-relativistic quantum electrodynamics, J.Phys.A.Math.Gen.16,(1983)49-70.

[4] A.Arai, Rigorous theory of spectra and radiation for a model in a quantum electrodynamics, J.Math.Phys.24(1983)1896-1910.

[5] A.Arai, An asymptotic analysis and its applications to the nonrelativistic limit of the Pauli-Fierz and a spin-boson model, J.Math.Phys.31(1990)2653-2663.

[6] F.Hiroshima, Scaling limit of a model in quantum electrodynamics, J.Math.Phys.34(1993)4478-4578.

[7] F.Hiroshima, Diamagnetic inequalities for a systems of nonrelativistic particles with a quantized radiation field. to appear Rev.Math.Phys.Vol.8 No.2.

[8] F.Hiroshima, Functional integral representation of a model in quantum electrodynamics, submitted to J.Funct.Anal..

[9] E.C.Titchmarsh, Theory of Fourier Integrals, 2nd.ed.(Oxford Univ.Press, Oxford,1948). [10] M.Reed, B.Simon, Method of Modern Mathematical Physics II Fourier Analysis and 
Self-Adjoint operator, Academic Press(1975).

[11] E.A. Berezin, "Second quantization", Academic Press(1966).

[12] E.B.Davies, Properties of the Green's functions of some Schrödinger operators, J.London Math.Soc.7,483-493(1976). 